\title{
The $k$-cut model in deterministic and random trees
}

\author{
Gabriel Berzunza \\ Department of Mathematical Sciences \\ University of Liverpool \\ Liverpool, U.K. \\ gabriel.berzunza-ojeda@liverpool.ac.uk \\ Xing Shi Cai Cecilia Holmgren* \\ Mathematics Department \\ Uppsala University \\ Uppsala, Sweden \\ \{xingshi.cai, cecilia.holmgren\}@math.uu.se
}

Submitted: Apr 1, 2020; Accepted: Nov 27, 2020; Published: Jan 29, 2021

(C) The authors. Released under the CC BY-ND license (International 4.0).

\begin{abstract}
The $k$-cut number of rooted graphs was introduced by Cai et al. as a generalization of the classical cutting model by Meir and Moon. In this paper, we show that all moments of the $k$-cut number of conditioned Galton-Watson trees converge after proper rescaling, which implies convergence in distribution to the same limit law regardless of the offspring distribution of the trees. This extends the result of Janson. Using the same method, we also show that the $k$-cut number of various random or deterministic trees of logarithmic height converges in probability to a constant after rescaling, such as random split-trees, uniform random recursive trees, and scale-free random trees.
\end{abstract}

Mathematics Subject Classifications: 60C05, 60F05, 05C05

\section{Introduction and main result}

In order to measure the difficulty for the destruction of a resilient network Cai et al. [12] introduced a generalization of the cut model of Meir and Moon [30] where each vertex (or edge) needs to be cut $k \in \mathbb{N}$ times (instead of only once) before it is destroyed. More

*This work is supported by the Knut and Alice Wallenberg Foundation, the Swedish Research Council and The Swedish Foundations' starting grant from Ragnar Söderbergs Foundation. 
precisely, consider that the resilient network is a rooted tree $\mathbb{T}_{n}$, with $n \in \mathbb{N}$ vertices. We assume that sibling vertices in $\mathbb{T}_{n}$ are ordered. (Such trees sometimes are referred to as plane trees.) We destroy it by removing its vertices as follows: Step 1: Choose a vertex uniformly at random from the component that contains the root and cut the selected vertex once. Step 2: If this vertex has been cut $k$ times, remove the vertex together with the edges attached to it from the tree. Step 3: If the root has been removed, then stop. Otherwise, go to step Step 1 . We let $\mathcal{K}_{k}\left(\mathbb{T}_{n}\right)$ denote the (random) total number of cuts needed to end this procedure the $k$-cut number, i.e., $\mathcal{K}_{k}\left(\mathbb{T}_{n}\right)$ models how much effort it takes to destroy the network. (For simplicity, we will omit the subscript $k$ and write $\mathcal{K}\left(\mathbb{T}_{n}\right)$.) It should be clear that one can define analogously an edge deletion version of the previous algorithm, where one needs to cut an edge $k$ times before removing it from the root component. Then, one would be interested in the number $\mathcal{K}_{e}\left(\mathbb{T}_{n}\right)$ of edge cuts needed to isolate the root of $\mathbb{T}_{n}$.

The case $k=1$ (i.e., the traditional cutting model of Meir and Moon [30]) has been well-studied by several authors. More precisely, Meir and Moon estimated the first and second moment of the 1-cut number in the cases when $\mathbb{T}_{n}$ is a Cayley tree [30] and a recursive tree [31]. Subsequently, several weak limit theorems for the 1-cut number have been obtained for Cayley trees (Panholzer [33, 34]), complete binary trees (Janson [24]), conditioned Galton-Watson trees (Janson [25] and Addario-Berry et al. [1]), recursive trees (Drmota et al. [16], Iksanov and Möhle [23]), binary search trees (Holmgren [19]) and split trees (Holmgren [20]). In the general case $k \geqslant 1$, the authors in [12] established first moment estimates of $\mathcal{K}\left(\mathbb{T}_{n}\right)$ for families of deterministic and random trees, such as paths, complete binary trees, split trees, random recursive trees and conditioned GaltonWatson trees. In particular, the authors in [12] have proven a weak limit theorem for $\mathcal{K}\left(\mathbb{T}_{n}\right)$ when $\mathbb{T}_{n}$ is a path consisting of $n$ vertices. More recently, Cai and Holmgren [11] also obtained a weak limit theorem in the case when $\mathbb{T}_{n}$ is a complete binary tree.

In this work, we continue the investigation of this general cutting-down procedure in conditioned Galton-Watson trees and show that $\mathcal{K}\left(\mathbb{T}_{n}\right)$, after a proper rescaling, converges in distribution to a non-degenerate random variable. More precisely, let $\xi$ be a nonnegative integer-valued random variable such that

$$
\mathbb{E}[\xi]=1 \quad \text { and } \quad 0<\sigma^{2}:=\operatorname{Var}(\xi)<\infty .
$$

We further assume that the distribution of $\xi$ is aperiodic. This last condition is to avoid unnecessary complications, but our results can be extended to the periodic case. We then consider a Galton-Watson process with (critical) offspring distribution $\xi$. Let $\mathbb{T}_{n}$ be the family tree conditioned on its number of vertices being $n \in \mathbb{N}$, providing that this conditioning makes sense. The main result of this paper is the following. We write $\stackrel{d}{\rightarrow}$ to denote convergence in distribution. (In the rest of the paper CRT stands for Continuum Random Tree.)

Theorem 1. Let $k \in \mathbb{N}$. Let $\mathbb{T}_{n}$ be a Galton-Watson tree conditioned on its number of vertices being $n \in \mathbb{N}$ with offspring distribution $\xi$ satisfying (1). Then,

$$
\sigma^{-1 / k} n^{-1+1 / 2 k} \mathcal{K}\left(\mathbb{T}_{n}\right) \stackrel{d}{\rightarrow} Z_{\mathrm{CRT}}, \quad \text { as } n \rightarrow \infty
$$


where $Z_{\mathrm{CRT}}$ is a non-degenerate random variable whose law is determined entirely by its moments: $\mathbb{E}\left[Z_{\mathrm{CRT}}^{0}\right]=1$, and for $q \in \mathbb{N}, \mathbb{E}\left[Z_{\mathrm{CRT}}^{q}\right]=\eta_{k, q}$ with

$$
\eta_{k, q}:=q ! \int_{0}^{\infty} \cdots \int_{0}^{\infty} y_{1}\left(y_{1}+y_{2}\right) \cdots\left(y_{1}+\cdots+y_{q}\right) e^{-\frac{\left(y_{1}+\cdots+y_{q}\right)^{2}}{2}} F_{q}\left(\mathbf{y}_{q}\right) \mathrm{d} y_{q} \cdots \mathrm{d} y_{1},
$$

where $\mathbf{y}_{q}=\left(y_{1}, \ldots, y_{q}\right) \in \mathbb{R}_{+}^{q}$ and

$$
F_{q}\left(\mathbf{y}_{q}\right):=\int_{0}^{\infty} \int_{0}^{x_{1}} \cdots \int_{0}^{x_{q-1}} \exp \left(-\frac{y_{1} x_{1}^{k}+y_{2} x_{2}^{k}+\cdots+y_{q} x_{q}^{k}}{k !}\right) \mathrm{d} x_{q} \cdots \mathrm{d} x_{2} \mathrm{~d} x_{1} .
$$

Furthermore, if $\mathbb{E}\left[\xi^{p}\right]<\infty$ for every $p \in \mathbb{Z}_{\geqslant 0}$, then for every $q \in \mathbb{Z}_{\geqslant 0}$,

$$
\sigma^{-q / k} n^{-q+q / 2 k} \mathbb{E}\left[\mathcal{K}\left(\mathbb{T}_{n}\right)^{q}\right] \rightarrow \mathbb{E}\left[Z_{\mathrm{CRT}}^{q}\right], \quad \text { as } n \rightarrow \infty .
$$

In the case $k=1$, Theorem 1 reduces to a $Z_{\mathrm{CRT}}$ having a Rayleigh distribution with density $x e^{-x^{2} / 2}$, for $x \in \mathbb{R}_{+}$. More precisely, one can verify that $\eta_{1, q}=2^{q / 2} \Gamma(1+q / 2)$, for $q \in \mathbb{Z}_{\geqslant 0}$, which are the moments of a random variable with the Rayleigh distribution; in this paper $\Gamma(\cdot)$ denotes the well-known gamma function. As we mentioned earlier, the case $k=1$ has been shown in [25, Theorem 1.6] (or Addario-Berry et al. [1]). We henceforth assume throughout this paper that $k \geqslant 2$.

It is also important to mention that we could not find a simpler expression (in general) for the moments $\eta_{k, q}$ except for some particular instances. For $q=1$, we have

$$
\eta_{k, 1}=2^{-\frac{1}{2 k}} \frac{(k !)^{\frac{1}{k}}}{k} \Gamma\left(\frac{1}{k}\right) \Gamma\left(1-\frac{1}{2 k}\right) .
$$

Then Theorem 1 provides a proof of [12, Lemma 4.10], where an estimation of the first moment of $\mathcal{K}\left(\mathbb{T}_{n}\right)$ was first announced but whose proof was left to the reader. One can also compute with the help of Mathematica the second moment of $Z_{\mathrm{CRT}}$ or other particular examples. However, the expressions are too involved and we decided not to include them.

On the other hand, let $\left(U_{1}, \ldots, U_{q}\right)$ be $q$ i.i.d. leaves of a Brownian CRT and define the vector $\left(L_{0}^{\mathrm{CRT}}, L_{1}^{\mathrm{CRT}}, \ldots, L_{q}^{\mathrm{CRT}}\right)$ where $L_{0}^{\mathrm{CRT}}=0$ and $L_{i}^{\mathrm{CRT}}$ is the total length of the minimal subtree of a Brownian CRT which connects its root and the leaves of $U_{1}, \ldots, U_{i}$; see [3, Lemma 21] from where one can deduce explicitly the distribution of $\left(L_{0}^{\mathrm{CRT}}, L_{1}^{\mathrm{CRT}}, \ldots L_{q}^{\mathrm{CRT}}\right)$. From the proof of Theorem 1 , we obtain, for $q \in \mathbb{N}$, that

$$
\eta_{k, q}=q ! \int_{0}^{\infty} \int_{0}^{x_{1}} \cdots \int_{0}^{x_{q-1}} \mathbb{E}\left[\exp \left(-\frac{\sum_{i=1}^{q}\left(L_{i}^{\mathrm{CRT}}-L_{i-1}^{\mathrm{CRT}}\right) x_{i}^{k}}{k !}\right)\right] \mathrm{d} \overleftarrow{\mathbf{x}}_{q},
$$

where $\overleftarrow{\mathbf{x}}_{q}=\left(x_{q}, \ldots, x_{1}\right) \in \mathbb{R}_{+}^{q}$. This suggests that it ought to be possible to build the random variable $Z_{\mathrm{CRT}}$ by some construction that can be interpreted as the $k$-cut model on the Brownian CRT defined by Aldous $[2,3]$. The appearance of the Brownian CRT in this framework should not come as a surprise since it is well-known that if we assign length $n^{-1 / 2}$ to each edge of the Galton-Watson tree $\mathbb{T}_{n}$, then the latter converges weakly 
to a Brownian CRT as $n \rightarrow \infty$. We believe that this connection can be exploited even more than the one used in this work in order to obtain the precise distribution of $Z_{\mathrm{CRT}}$. For example, ideas from [6] and [1] could be useful to answer this question.

The approach used in this work consists of implementing an extension of the idea of Janson [25], which was used in [12], in order to study the $k$-cut model on deterministic and random trees. The authors in [12] introduced an equivalent model that allows them to define $\mathcal{K}\left(\mathbb{T}_{n}\right)$ in terms of the number of records in $\mathbb{T}_{n}$ when vertices are assigned random labels. More precisely, let $\left(E_{i, v}\right)_{i \geqslant 1, v \in \mathbb{T}_{n}}$ be a sequence of independent exponential random variables with parameter $1 ; \operatorname{Exp}(1)$ for short. Let $G_{r, v}:=\sum_{1 \leqslant i \leqslant r} E_{i, v}$, for $r \in \mathbb{N}$ and $v \in \mathbb{T}_{n}$. Clearly, $G_{r, v}$ has a gamma distribution with parameters $(r, 1)$, which we denote by $\operatorname{Gamma}(r)$. Imagine that each vertex $v \in \mathbb{T}_{n}$ has an alarm clock and $v$ 's clock fires at times $\left(G_{r, v}\right)_{r \geqslant 1}$. If we cut a vertex when its alarm clock fires, then due to the memoryless property of exponential random variables, we are actually choosing a vertex uniformly at random to cut. However, this also means that we are cutting vertices that have already been removed from the tree. Thus, for a cut on vertex $v$ at time $G_{r, v}$ (for some $r \in\{1, \ldots, k\})$ to be counted in $\mathcal{K}\left(\mathbb{T}_{n}\right)$, none of its strict ancestors can already have been cut $k$ times, i.e.,

$$
G_{r, v}<\min \left\{G_{k, u}: u \in \mathbb{T}_{n} \text { and } u \text { is a strict ancestor of } v\right\} .
$$

When the previous event happens, we say that $G_{r, v}$, or simply $v$, is an $r$-record and let

$$
I_{r, v}:=\llbracket G_{r, v}<\min \left\{G_{k, u}: u \in \mathbb{T}_{n} \text { and } u \text { is a strict ancestor of } v\right\} \rrbracket,
$$

where $\llbracket \cdot \rrbracket$ denotes the Iverson bracket, i.e., $\llbracket S \rrbracket=1$ if the statement $S$ is true and $\llbracket S \rrbracket=0$ otherwise. Let $\mathcal{K}_{r}\left(\mathbb{T}_{n}\right)$ be the number of $r$-records, i.e., $\mathcal{K}_{r}\left(\mathbb{T}_{n}\right):=\sum_{v \in \mathbb{T}_{n}} I_{r, v}$. Then, it should be clear that

$$
\mathcal{K}\left(\mathbb{T}_{n}\right) \stackrel{d}{=} \sum_{1 \leqslant r \leqslant k} \mathcal{K}_{r}\left(\mathbb{T}_{n}\right)
$$

where $\stackrel{d}{=}$ denotes equal in distribution.

Loosely speaking, we then consider the well-known depth-first search walk or contour function $V_{n}=\left(V_{n}(t), t \in[0,2(n-1)]\right)$ of the (ordered) tree $\mathbb{T}_{n}$ as depicted in Figure 1 , that is, $V_{n}(t)$ is "the depth of the $t$-th vertex" visited in this walk; this will be made precise in the next section. As it is well-known (see Aldous [3, Theorem 23 with Remark 2] or $\left[29\right.$, Theorem 1]), when $\mathbb{T}_{n}$ is a conditioned Galton-Watson with offspring distribution satisfying (1), we have that

$$
\left(n^{-1 / 2} V_{n}(2(n-1) t), t \in[0,1]\right) \stackrel{d}{\rightarrow} 2 \sigma^{-1} B^{\mathrm{ex}}, \quad \text { as } n \rightarrow \infty,
$$

in $C\left([0,1], \mathbb{R}_{+}\right)$, with its usual topology, and where $B^{\text {ex }}=\left(B^{\operatorname{ex}}(t), t \in[0,1]\right)$ is a standard normalized Brownian excursion. It has been shown in $\left[12\right.$, Lemma 2.1] that $^{1}$

\footnotetext{
${ }^{1}$ For two sequences of non-negative real numbers $\left(A_{n}\right)_{n \geqslant 1}$ and $\left(B_{n}\right)_{n \geqslant 1}$ such that $B_{n}>0$, we write $A_{n} \sim B_{n}$ if $A_{n} / B_{n} \rightarrow 1$ as $n \rightarrow \infty$
} 

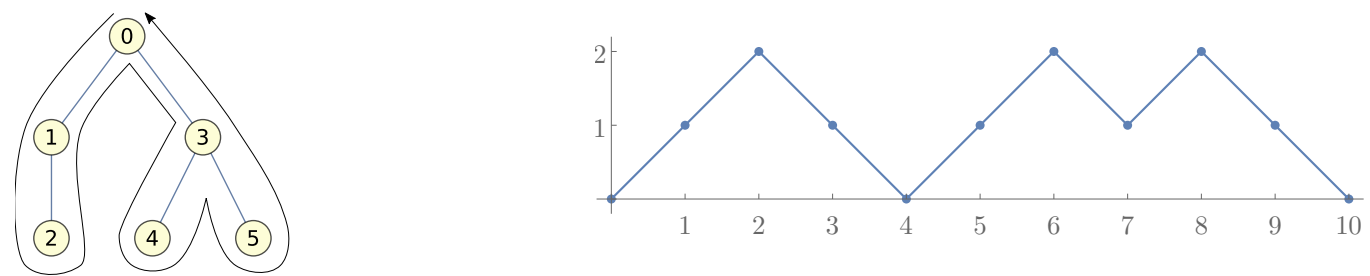

Figure 1: An example of a depth-first search walk in a tree and the corresponding $V_{n}$.

$\mathbb{E}\left[I_{r, v}\right] \sim C_{r, k} d_{n}(v)^{-r / k}$, for some (explicit) constant $C_{r, k}>0$, where $d_{n}(v)$ is the depth of the vertex $v \in \mathbb{T}_{n}$. Let $\circ$ denote the root of $\mathbb{T}_{n}$. Thus, heuristically

$$
\begin{aligned}
\mathbb{E}\left[\mathcal{K}_{r}\left(\mathbb{T}_{n}\right) \mid \mathbb{T}_{n}\right] & \approx \sum_{v \in \mathbb{T}_{n} \backslash\{\circ\}} \frac{C_{r, k}}{d_{n}(v)^{r / k}} \approx \frac{C_{r, k}}{2} \int_{0}^{2(n-1)} \frac{\mathrm{d} t}{V_{n}(t)^{r / k}} \\
& \approx \frac{C_{r, k}}{n^{-1+\frac{r}{2 k}}} \int_{0}^{1}\left(\frac{V_{n}(2(n-1) t)}{\sqrt{n}}\right)^{-\frac{r}{k}} \mathrm{~d} t \\
& \approx \frac{C_{r, k}}{n^{-1+\frac{r}{2 k}}}\left(\frac{\sigma}{2}\right)^{\frac{r}{k}} \int_{0}^{1} \frac{\mathrm{d} t}{B^{\operatorname{ex}}(t)^{r / k}},
\end{aligned}
$$

in distribution, as $n \rightarrow \infty$. (The reader should bear in mind that the above calculation is not rigorous at all and that the purpose is only to illustrate the idea of proof.) One then expects that

$$
\sigma^{-r / k} n^{-1+\frac{r}{2 k}} \mathbb{E}\left[\mathcal{K}_{r}\left(\mathbb{T}_{n}\right)\right] \sim C_{r, k} \mathbb{E}\left[\int_{0}^{1}\left(2 B^{\operatorname{ex}}(t)\right)^{-r / k} \mathrm{~d} t\right], \quad \text { as } n \rightarrow \infty,
$$

which coincides with the right-hand side of (3) when $r=q=1$. Note that this informal computation suggests that ${ }^{2} \mathbb{E}\left[\mathcal{K}_{r}\left(\mathbb{T}_{n}\right)\right]=O\left(n^{1-\frac{r}{2 k}}\right)$, for $r \in\{1, \ldots, k\}$. As a consequence, Markov's inequality implies that $n^{-1+\frac{1}{2 k}} \mathcal{K}_{r}\left(\mathbb{T}_{n}\right) \rightarrow 0$ in probability, as $n \rightarrow \infty$, for $r \in$ $\{2, \ldots, k\}$. As shown later, by the identity in (5), it would be enough to prove Theorem 1 for $\mathcal{K}_{1}\left(\mathbb{T}_{n}\right)$ instead of $\mathcal{K}\left(\mathbb{T}_{n}\right)$.

In the rest of the paper, Section 2 and Section 3 make the above argument precise and extend it to higher moments. This will allow us to use the method of moments for proving Theorem 1. In Section 4, we also apply the same idea to get all moments of the number of records in paths and several types of trees of logarithmic height, e.g., complete binary trees, split trees, uniform random recursive trees and scale-free trees.

\section{Preliminary results}

The purpose of this section is to establish a general convergence result for the number of 1-records $\mathcal{K}_{1}\left(\mathbb{T}_{n}\right)$ of a deterministic rooted ordered tree $\mathbb{T}_{n}$. The results of this section

\footnotetext{
${ }^{2}$ For two sequences of non-negative real numbers $\left(A_{n}\right)_{n \geqslant 1}$ and $\left(B_{n}\right)_{n \geqslant 1}$ such that $B_{n}>0$, we write $A_{n}=O\left(B_{n}\right)$ if $\lim \sup _{n \rightarrow \infty} A_{n} / B_{n}<\infty$.
} 
can also be viewed as a generalization of those in Janson [25] and in Cai, et al. [12]. Furthermore, these results will allow us to study the convergence of $\mathcal{K}\left(\mathbb{T}_{n}\right)$ not only for conditioned Galton-Watson trees, but also for other classes of random trees in Section 4. We start by defining a probability measure through a continuous function in the same spirit as in [25, Theorem 1.9]. Let $I \subseteq \mathbb{R}_{+}$be an interval. For a function $f: I \rightarrow \mathbb{R}_{+}$and $t_{1}, \ldots, t_{q} \in I$ with $q \in \mathbb{N}$, we define

$$
L_{f}\left(t_{1}, \ldots, t_{q}\right):=\sum_{i=1}^{q} f\left(t_{(i)}\right)-\sum_{i=1}^{q-1} \inf _{t \in\left[t_{(i)}, t_{(i+1)}\right]} f(t),
$$

where $t_{(1)}, \ldots, t_{(q)}$ are $t_{1}, \ldots, t_{q}$ arranged in nondecreasing order. Notice that $L_{f}\left(t_{1}, \ldots, t_{q}\right)$ is symmetric in $t_{1}, \ldots, t_{q}$ and that $L_{f}(t)=f(t)$ for $t \in I$. Define

$$
D_{f}\left(t_{1}\right):=L_{f}\left(t_{1}\right) \text { and } D_{f}\left(t_{1}, \ldots, t_{q}\right):=L_{f}\left(t_{1}, \ldots, t_{q}\right)-L_{f}\left(t_{1}, \ldots, t_{q-1}\right) \text {, for } q \geqslant 2 \text {. }
$$

We also consider the functional

$$
G_{f}\left(\mathbf{t}_{q}, \mathbf{x}_{q}\right):=\exp \left(-\frac{D_{f}\left(t_{1}\right) x_{1}^{k}+\cdots+D_{f}\left(t_{1}, \ldots, t_{q}\right) x_{q}^{k}}{k !}\right),
$$

for $\mathbf{x}_{q}=\left(x_{1}, \ldots, x_{q}\right) \in \mathbb{R}_{+}^{q}$ and $\mathbf{t}_{q}=\left(t_{1}, \ldots, t_{q}\right) \in I^{q}$. If $I=[0,1]$, we further define, for $q \in \mathbb{N}, m_{0}(f):=1$ and

$$
m_{q}(f):=q ! \int_{0}^{1} \int_{0}^{1} \cdots \int_{0}^{1} \int_{0}^{\infty} \int_{0}^{x_{1}} \cdots \int_{0}^{x_{q-1}} G_{f}\left(\mathbf{t}_{q}, \mathbf{x}_{q}\right) \mathrm{d} \overleftarrow{\mathbf{x}}_{q} \mathrm{~d} \overleftarrow{\mathbf{t}}_{q}, \quad \text { for } q \geqslant 2,
$$

where $\overleftarrow{\mathbf{x}}_{q}=\left(x_{q}, \ldots, x_{1}\right)$ and $\overleftarrow{\mathbf{t}}_{q}=\left(t_{q}, \ldots, t_{1}\right)$.

Theorem 2. Let $k \in \mathbb{N}$. Suppose that $f \in C\left([0,1], \mathbb{R}_{+}\right)$is such that $\int_{0}^{1} f(t)^{-1 / k} \mathrm{~d} t<\infty$. Then there exists a unique probability measure $\nu_{f}$ on $[0, \infty)$ with finite moments given by

$$
\int_{[0, \infty)} x^{q} \nu_{f}(\mathrm{~d} x)=m_{q}(f), \quad \text { for } q \in \mathbb{Z}_{\geqslant 0}
$$

Proof. We only prove uniqueness here. The proof for existence follows along the lines of [25, Proof of Theorem 1.9, Pages 18-19] and details are left to the interested reader. Informally speaking, the idea in [25] for the proof of existence is to build a sequence of functions that satisfy the conditions of Lemma 3 below. Define the function

$$
H_{f, q}\left(\mathbf{t}_{q}\right):=\int_{0}^{\infty} \int_{0}^{x_{1}} \cdots \int_{0}^{x_{q-1}} G_{f}\left(\mathbf{t}_{q}, \mathbf{x}_{q}\right) \mathrm{d} \overleftarrow{\mathbf{x}}_{q} .
$$

By changing the order of integration, we obtain that

$$
H_{f, q}\left(\mathbf{t}_{q}\right)=\int_{0}^{\infty} \int_{x_{q}}^{\infty} \cdots \int_{x_{2}}^{\infty} G_{f}\left(\mathbf{t}_{q}, \mathbf{x}_{q}\right) \mathrm{d} \mathbf{x}_{q}
$$


for $\mathbf{x}_{q}=\left(x_{1}, \ldots, x_{q}\right) \in \mathbb{R}_{+}^{q}$ and $\mathbf{t}_{q}=\left(t_{1}, \ldots, t_{q}\right) \in[0,1]^{q}$. By making the change of variables $x_{q}=w_{q}, x_{q-1}=w_{q}+w_{q-1}, \ldots, x_{1}=w_{q}+\cdots+w_{1}$, we see that

$$
H_{f, q}\left(\mathbf{t}_{q}\right)=\int_{[0, \infty)^{q}} \exp \left(-\frac{1}{k !} \sum_{i=1}^{q} D_{f}\left(t_{1}, \ldots, t_{i}\right)\left(\sum_{j=i}^{q} w_{j}\right)^{k}\right) \mathrm{d} \mathbf{w}_{q}
$$

where $\mathbf{w}_{q}=\left(w_{1}, \ldots, w_{q}\right) \in \mathbb{R}_{+}^{q}$. From the inequality $\left(x_{1}+\cdots+x_{q}\right)^{k} \geqslant x_{1}^{k}+\cdots+x_{q}^{k}$, we observe that

$$
\begin{aligned}
H_{f, q}\left(\mathbf{t}_{q}\right) & \leqslant \prod_{j=1}^{q} \int_{0}^{\infty} \exp \left(-\frac{w_{j}^{k}}{k !} \sum_{i=1}^{j} D_{f}\left(t_{1}, \ldots, t_{i}\right)\right) \mathrm{d} w_{j} \\
& =\Gamma(1+1 / k)^{q} \Gamma(1+k)^{q / k} \prod_{j=1}^{q}\left(\sum_{i=1}^{j} D_{f}\left(t_{1}, \ldots, t_{i}\right)\right)^{-1 / k} \\
& =\Gamma(1+1 / k)^{q} \Gamma(1+k)^{q / k} \prod_{i=1}^{q} L_{f}\left(t_{1}, \ldots, t_{i}\right)^{-1 / k} \\
& \leqslant \Gamma(1+1 / k)^{q} \Gamma(1+k)^{q / k} \prod_{i=1}^{q} f\left(t_{i}\right)^{-1 / k}
\end{aligned}
$$

where for the last inequality we have used the fact that $L_{f}\left(t_{1}, \ldots, t_{i}\right) \geqslant \max _{1 \leqslant j \leqslant i} f\left(t_{j}\right)$, for $1 \leqslant i \leqslant q$. The later follows from the symmetry of $L_{f}$; see [25, Lemma 4.1] for a proof. Then, the previous inequality allows us to conclude that

$$
0 \leqslant m_{q}(f) \leqslant q ! \Gamma(1+1 / k)^{q} \Gamma(1+k)^{q / k}\left(\int_{0}^{1} f(t)^{-1 / k} \mathrm{~d} t\right)^{q} .
$$

We conclude that there exists $a>0$ such that $\sum_{q=0}^{\infty} m_{q}(f) \frac{x^{q}}{q !}<\infty$, for $0 \leqslant x<a$. Then a probability measure with moments $m_{q}(f)$ has a finite generating function in a neighbourhood of 0 . Thus, it is well-known that this implies that the probability measure is unique; see, e.g., [18, Section 4.10].

Consider a rooted ordered tree $\mathbb{T}_{n}$ with root $\circ$ and $n \in \mathbb{N}$ vertices. We now explain how $\mathbb{T}_{n}$ can be encoded by a continuous function. We define the so-called depth-first search function [2, page 260], $\psi_{n}:\{0,1, \ldots, 2(n-1)\} \rightarrow\left\{\right.$ vertices of $\left.\mathbb{T}_{n}\right\}$ such that $\psi_{n}(i)$ is the $(i+1)$-th vertex visited in a depth-first walk on the tree starting from the root $\circ$. Note that $\psi_{n}(i)$ and $\psi_{n}(i+1)$ always are neighbours, and thus, we extend $\psi$ to [0,2(n-1)] by letting, for $1 \leqslant i<t<i+1 \leqslant 2(n-1), \psi_{n}(t)$ to be the one of $\psi_{n}(i)$ and $\psi_{n}(i+1)$ that has largest depth (recall that the depth of a vertex $v \in \mathbb{T}_{n}$ is the distance, i.e., number of edges, between $\circ$ to $v)$. Let $d_{n}(v)$ be the depth of a vertex $v \in \mathbb{T}_{n}$. We further define the depth-first walk $V_{n}$ of $\mathbb{T}_{n}$ by

$$
V_{n}(i):=d_{n}(\psi(i)), \quad i \in\{0, \ldots, 2(n-1)\},
$$


and extend $V_{n}$ to $[0,2(n-1)]$ by linear interpolation. Thus $V_{n} \in C\left([0,2(n-1)], \mathbb{R}_{+}\right)$. See Figure 1 for an example of $V_{n}$. Furthermore, we normalize the domain of $V_{n}$ to $[0,1]$ by defining

$$
\widetilde{V}_{n}(t):=V_{n}(2(n-1) t) \text { and } \widehat{V}_{n}(t):=\left\lceil V_{n}(2(n-1) t)\right\rceil,
$$

for $t \in[0,1]$. Thus $\widetilde{V}_{n} \in C\left([0,1], \mathbb{R}_{+}\right)$. Note that $d_{n}(\psi(t))=\left\lceil V_{n}(t)\right\rceil$, for $t \in[0,2(n-1)]$. Moreover,

$$
\max _{v \in \mathbb{T}_{n}} d_{n}(v)=\sup _{t \in[0,2(n-1)]} V_{n}(t)=\sup _{t \in[0,1]} \widetilde{V}_{n}(t) .
$$

We now state the central result of this section, that is, a general limit theorem in distribution for the number of 1 -records $\mathcal{K}_{1}\left(\mathbb{T}_{n}\right)$ of a deterministic rooted tree $\mathbb{T}_{n}$ with $n$ vertices. It is important to notice that $\mathcal{K}_{1}\left(\mathbb{T}_{n}\right)$ is a random variable since the 1 -records are random. From now on, we always assume that $k \geqslant 2$.

Lemma 3. Suppose that $\left(\mathbb{T}_{n}\right)_{n \geqslant 1}$ is a sequence of ordered (deterministic) rooted trees, and denote the corresponding normalized depth-first walks by $\widetilde{V}_{n}$ and $\widehat{V}_{n}$. Suppose that there exists a sequence $\left(a_{n}\right)_{n \geqslant 1}$ of non-negative real numbers with $\lim _{n \rightarrow \infty} a_{n}=0, \lim _{n \rightarrow \infty} n a_{n}^{1 / k}=$ $\infty$ and a function $f \in C\left([0,1], \mathbb{R}_{+}\right)$such that

(a) $a_{n} \widetilde{V}_{n}(t) \rightarrow f(t)$, in $C\left([0,1], \mathbb{R}_{+}\right)$, as $n \rightarrow \infty$.

(b) $\int_{0}^{1}\left(a_{n} \widehat{V}_{n}(t)\right)^{-1 / k} \mathrm{~d} t \rightarrow \int_{0}^{1} f(t)^{-1 / k} \mathrm{~d} t<\infty$, as $n \rightarrow \infty$.

Then, for each $q \in \mathbb{Z}_{\geqslant 0}$,

$$
n^{-q} a_{n}^{-q / k} \mathbb{E}\left[\mathcal{K}_{1}\left(\mathbb{T}_{n}\right)^{q}\right] \rightarrow m_{q}(f),
$$

as $n \rightarrow \infty$, where $m_{q}(f)$ is defined in $(9)$. Moreover, $n^{-1} a_{n}^{-1 / k} \mathcal{K}_{1}\left(\mathbb{T}_{n}\right) \stackrel{d}{\rightarrow} Z_{f}$, as $n \rightarrow \infty$, where $Z_{f}$ is a random variable with distribution $\nu_{f}$ defined by Theorem 2.

Before proving Lemma 3, we need to establish some preliminary results and to introduce some further notation. For $q \in \mathbb{N}$ and vertices $v_{1}, \ldots, v_{q} \in \mathbb{T}_{n}$, let $L_{n}\left(v_{1}, \ldots, v_{q}\right)$ be the number of edges in the subtree of $\mathbb{T}_{n}$ spanned by $v_{1}, \ldots, v_{q}$ and its root $\circ$ (i.e., the minimal number of edges that are needed to connect $v_{1}, \ldots, v_{q}$ and $\left.\circ\right)$. We write $D_{n}\left(v_{1}\right):=L_{n}\left(v_{1}\right)$ and $D_{n}\left(v_{1}, \ldots, v_{q}\right):=L_{n}\left(v_{1}, \ldots, v_{q}\right)-L_{n}\left(v_{1}, \ldots, v_{q-1}\right)$ for $q \geqslant 2$. We also consider the functional

$$
G_{n}\left(\mathbf{v}_{q}, \mathbf{x}_{q}\right):=\exp \left(-\frac{D_{n}\left(v_{1}\right) x_{1}^{k}+\cdots+D_{n}\left(v_{1}, \ldots, v_{q}\right) x_{q}^{k}}{k !}\right),
$$

for $\mathbf{x}_{q}=\left(x_{1}, \ldots, x_{q}\right) \in \mathbb{R}_{+}^{q}$ and $\mathbf{v}_{q}=\left(v_{1}, \ldots, v_{q}\right) \in \mathbb{T}_{n}^{q}$. We denote by $\Gamma(k, \cdot)$ the upper incomplete gamma function of parameter $k \in \mathbb{N}$, i.e.,

$$
\Gamma(k, x)=\int_{x}^{\infty} t^{k-1} e^{-t} \mathrm{~d} t, \quad \text { for } x \geqslant 0 .
$$


Remark 4. Let $\mathbb{T}_{n}$ be an ordered (deterministic) rooted tree with depth-first search walk $\psi_{n}$ and the corresponding function $V_{n}$. It is not difficult to see that $L_{n}$ and $L_{\left\lceil V_{n}\right\rceil}$ are connected, in the sense that $L_{n}\left(\psi_{n}\left(t_{1}\right), \ldots, \psi_{n}\left(t_{q}\right)\right)=L_{\left\lceil V_{n}\right\rceil}\left(t_{1}, \ldots, t_{q}\right)$ for $t_{1}, \ldots, t_{q} \in$ $[0,2(n-1)]$; see [25, Lemma 4.4] for a proof of this fact.

Lemma 5. Let $\mathbb{T}_{n}$ be an ordered (deterministic) rooted tree with $n \in \mathbb{N}$ vertices. Suppose that there exists a sequence $\left(a_{n}\right)_{n \geqslant 1}$ of non-negative real numbers such that $\lim _{n \rightarrow \infty} a_{n}=0$ and $\max _{v \in \mathbb{T}_{n}} d_{n}(v)=O\left(a_{n}^{-1}\right)$. Let $\alpha:=\frac{1}{2}\left(\frac{1}{k}+\frac{1}{k+1}\right)$ and $x_{0}:=a_{n}^{\alpha}$. Then, for $q \in \mathbb{N}$ and uniformly for all $x \in\left[0, x_{0}\right]$,

$$
\begin{aligned}
\mathbb{P}(\operatorname{Gamma}(k)>x)^{D_{n}\left(v_{1}, \ldots, v_{q}\right)} & =\left(\frac{\Gamma(k, x)}{\Gamma(k)}\right)^{D_{n}\left(v_{1}, \ldots, v_{q}\right)} \\
& =\left(1+O\left(a_{n}^{\frac{1}{2 k}}\right)\right) \exp \left(-\frac{D_{n}\left(v_{1}, \ldots, v_{q}\right) x^{k}}{k !}\right)
\end{aligned}
$$

where the vertices $v_{1}, \ldots, v_{q} \in \mathbb{T}_{n}$.

Proof. Our claim can be shown along the lines of [12, Proof of Lemma 5.1].

Recall that for two sequences of non-negative real numbers $\left(A_{n}\right)_{n \geqslant 1}$ and $\left(B_{n}\right)_{n \geqslant 1}$ such that $B_{n}>0$, one writes $A_{n}=o\left(B_{n}\right)$ if $\lim _{n \rightarrow \infty} A_{n} / B_{n}=0$.

Lemma 6. Let $\mathbb{T}_{n}$ be an ordered (deterministic) rooted tree with $n \in \mathbb{N}$ vertices. Suppose that there exists a sequence $\left(a_{n}\right)_{n \geqslant 1}$ of non-negative real numbers with $\lim _{n \rightarrow \infty} a_{n}=0$, $\lim _{n \rightarrow \infty} n a_{n}^{1 / k}=\infty$ and $\max _{v \in \mathbb{T}_{n}} d_{n}(v)=O\left(a_{n}^{-1}\right)$. Then the moments of $\mathcal{K}_{1}\left(\mathbb{T}_{n}\right)$ are given by

$$
n^{-q} a_{n}^{-q / k} \mathbb{E}\left[\mathcal{K}_{1}\left(\mathbb{T}_{n}\right)^{q}\right]=\left(1+O\left(a_{n}^{\frac{q}{2 k}}\right)\right) q ! \int_{0}^{1} \cdots \int_{0}^{1} \bar{H}_{n, q}\left(\mathbf{t}_{q}\right) \mathrm{d} \overleftarrow{\mathbf{t}}_{q}+o(1),
$$

where

$$
\bar{H}_{n, q}\left(\mathbf{t}_{q}\right):=\int_{0}^{\infty} \int_{0}^{x_{1}} \cdots \int_{0}^{x_{q-1}} G_{a_{n} \widehat{V}_{n}}\left(\mathbf{t}_{q}, \mathbf{x}_{q}\right) \exp \left(-a_{n}^{1 / k} \sum_{i=1}^{q} x_{i}\right) \mathrm{d} \overleftarrow{\mathbf{x}}_{q}, \quad \text { for } q \in \mathbb{N} .
$$

Proof. For simplicity, we write $X_{q}:=\mathcal{K}_{1}\left(\mathbb{T}_{n}\right)^{q}$ for $q \in \mathbb{Z}_{\geqslant 0}$ and note that $X_{q}=X_{1}^{q}$. For $q \in \mathbb{N}$, we observe that

$$
X_{q}=\left(X_{1}-1+1\right)^{q}=\left(X_{1}-1\right)^{q}+\sum_{p=0}^{q-1}\left(\begin{array}{l}
q \\
p
\end{array}\right)\left(X_{1}-1\right)^{p}=\left(X_{1}-1\right)^{q}+Y_{q} .
$$

where $Y_{q}:=\sum_{p=0}^{q-1} \sum_{l=0}^{p}\left(\begin{array}{l}q \\ p\end{array}\right)\left(\begin{array}{l}p \\ l\end{array}\right)(-1)^{p-l} X_{l}$. Recall that $I_{1, v}$ is the indicator that $v \in \mathbb{T}_{n}$ is a 1-record defined in (4). By the previous identity, we have that

$$
X_{q}=\sum_{v_{1}, \ldots, v_{q} \in \mathbb{T}_{n} \backslash\{0\}} I_{1, v_{1}} \cdots I_{1, v_{q}}+Y_{q}=q ! \sum_{v_{1}, \ldots, v_{q} \in \mathbb{T}_{n} \backslash\{0\}} \llbracket \mathcal{E}\left(v_{1}, \ldots, v_{q}\right) \rrbracket+Y_{q}
$$


where $\mathcal{E}\left(v_{1}, \ldots, v_{q}\right):=\left\{E_{1, v_{q}}<\cdots<E_{1, v_{1}}\right.$ and $v_{1}, \ldots, v_{q}$ are all 1-records $\}$; recall that $E_{1, v_{1}}, \ldots, E_{1, v_{q}}$ are independent random variables with an $\operatorname{Exp}(1)$ distribution. To see the last identity, note that each product $I_{1, v_{1}} \cdots I_{1, v_{q}}$ occurs $q$ ! times with indices permuted and for exactly one of these permutations we have that $E_{1, v_{q}}<\cdots<E_{1, v_{1}}$.

Consider the simple case $q=2$. Conditioning on $E_{1, v_{2}}=x_{2}<E_{1, v_{1}}=x_{1}$, we see that $v_{1}$ and $v_{2}$ are both 1-records, if and only if, the following two events happen:

(i) the $D_{n}\left(v_{1}\right)$ ancestors of $v_{1}$ are removed after time $x_{1}$;

(ii) the $D_{n}\left(v_{1}, v_{2}\right)$ vertices which are ancestors of $v_{2}$ but not of $v_{1}$ are removed after time $x_{2}$.

Since $x_{2}<x_{1}$, we note that the event (i) implies that the vertices which are both the ancestors of $v_{1}$ and $v_{2}$ are removed after $x_{1}$. Let $g(x):=\mathbb{P}(\operatorname{Gamma}(k)>x)$ for $x \in \mathbb{R}_{+}$. Since the events (i) and (ii) are independent, we have

$$
\mathbb{P}\left(\mathcal{E}\left(v_{1}, v_{2}\right)\right)=\int_{0}^{\infty} \int_{0}^{x_{1}} g\left(x_{1}\right)^{D_{n}\left(v_{1}\right)} g\left(x_{2}\right)^{D_{n}\left(v_{1}, v_{2}\right)} e^{-x_{1}-x_{2}} \mathrm{~d} x_{2} \mathrm{~d} x_{1} .
$$

Recall that we are assuming $k \geqslant 2$. Otherwise, when $k=1$, the above equality is not entirely correct since $\mathcal{E}\left(v_{1}, v_{2}\right)$ is impossible if $v_{2}$ is an ancestor of $v_{1}$; see [25, Lemma 4.3] for details in the case $k=1$.

By generalizing the previous argument to $q \in \mathbb{N}$, we see that

$$
\begin{aligned}
\mathbb{P}\left(\mathcal{E}\left(v_{1}, \ldots, v_{q}\right)\right)= & \int_{0}^{\infty} \int_{0}^{x_{1}} \cdots \int_{0}^{x_{q-1}} g\left(x_{1}\right)^{D_{n}\left(v_{1}\right)} \cdots g\left(x_{q}\right)^{D_{n}\left(v_{1}, \ldots, v_{q}\right)} e^{-\sum_{i=1}^{q} x_{i}} \mathrm{~d} \overleftarrow{\mathbf{x}}_{q} \\
= & \int_{0}^{x_{0}} \int_{0}^{x_{1}} \cdots \int_{0}^{x_{q-1}} g\left(x_{1}\right)^{D_{n}\left(v_{1}\right)} \cdots g\left(x_{q}\right)^{D_{n}\left(v_{1}, \ldots, v_{q}\right)} e^{-\sum_{i=1}^{q} x_{i}} \mathrm{~d} \overleftarrow{\mathbf{x}}_{q} \\
& +\int_{x_{0}}^{\infty} \int_{0}^{x_{1}} \cdots \int_{0}^{x_{q-1}} g\left(x_{1}\right)^{D_{n}\left(v_{1}\right)} \cdots g\left(x_{q}\right)^{D_{n}\left(v_{1}, \ldots, v_{q}\right)} e^{-\sum_{i=1}^{q} x_{i}} \mathrm{~d} \overleftarrow{\mathbf{x}}_{q} \\
= & A_{1}+A_{2},
\end{aligned}
$$

where $\overleftarrow{\mathbf{x}}_{q}=\left(x_{q}, \ldots, x_{1}\right) \in \mathbb{R}_{+}^{q}, x_{0}=a_{n}^{\alpha}$ and $\alpha=\frac{1}{2}\left(\frac{1}{k}+\frac{1}{k+1}\right)$. On the one hand, Lemma 5 implies that

$$
A_{2} \leqslant \int_{x_{0}}^{\infty} g(x)^{D_{n}\left(v_{1}\right)} e^{-x} \mathrm{~d} x \leqslant g\left(x_{0}\right)^{D_{n}\left(v_{1}\right)}=O\left(\exp \left(-\frac{x_{0}^{k}}{2 a_{n} k !}\right)\right)
$$

we have used our assumption $\max _{v \in \mathbb{T}_{n}} d_{n}(v)=O\left(a_{n}^{-1}\right)$. On the other hand, Lemma 5 also implies that

$$
\begin{aligned}
A_{1} & =\left(1+O\left(a_{n}^{\frac{1}{2 k}}\right)\right)^{q} \int_{0}^{x_{0}} \int_{0}^{x_{1}} \cdots \int_{0}^{x_{q-1}} G_{n}\left(\mathbf{v}_{q}, \mathbf{x}_{q}\right) e^{-\sum_{i=1}^{q} x_{i}} \mathrm{~d} \overleftarrow{\mathbf{x}}_{q} \\
& =\left(1+O\left(a_{n}^{\frac{q}{2 k}}\right)\right) \int_{0}^{\infty} \int_{0}^{x_{1}} \cdots \int_{0}^{x_{q-1}} G_{n}\left(\mathbf{v}_{q}, \mathbf{x}_{q}\right) e^{-\sum_{i=1}^{q} x_{i}} \mathrm{~d} \overleftarrow{\mathbf{x}}_{q}+A_{3},
\end{aligned}
$$


where $\mathbf{v}_{q}=\left(v_{1}, \ldots, v_{q}\right) \in \mathbb{T}_{n}^{q}$ and

$$
A_{3}=\left(1+O\left(a_{n}^{\frac{q}{2 k}}\right)\right) \int_{x_{0}}^{\infty} \int_{0}^{x_{1}} \cdots \int_{0}^{x_{q-1}} G_{n}\left(\mathbf{v}_{q}, \mathbf{x}_{q}\right) e^{-\sum_{i=1}^{q} x_{i}} \mathrm{~d} \overleftarrow{\mathbf{x}}_{q}=O\left(\exp \left(-\frac{x_{0}^{k}}{2 a_{n} k !}\right)\right)
$$

this estimation can be deduced similarly as the one for the integral $A_{2}$. Therefore, the previous estimations and Remark 4 allow us to conclude that $\mathbb{E}\left[X_{q}\right]$ equals to

$$
\begin{gathered}
\begin{array}{c}
\left(1+O\left(a_{n}^{\frac{q}{2 k}}\right)\right) q ! \sum_{v_{1}, \ldots, v_{q} \in \mathbb{T}_{n} \backslash\{0\}} \int_{0}^{\infty} \int_{0}^{x_{1}} \cdots \int_{0}^{x_{q-1}} G_{n}\left(\mathbf{v}_{q}, \mathbf{x}_{q}\right) e^{-\sum_{i=1}^{q} x_{i}} \mathrm{~d} \overleftarrow{\mathbf{x}}_{q} \\
+\mathbb{E}\left[Y_{q}\right]+o\left(n^{q} a_{n}^{q / k}\right) \\
=\left(1+O\left(a_{n}^{\frac{q}{2 k}}\right)\right) q ! 2^{-q} \int_{0}^{2(n-1)} \cdots \int_{0}^{2(n-1)} \int_{0}^{\infty} \int_{0}^{x_{1}} \cdots \int_{0}^{x_{q-1}} G_{\left\lceil V_{n}\right\rceil}\left(\mathbf{t}_{q}, \mathbf{x}_{q}\right) e^{-\sum_{i=1}^{q} x_{i}} \mathrm{~d} \overleftarrow{\mathbf{x}}_{q} \mathrm{~d} \overleftarrow{\mathbf{t}}_{q} \\
+\mathbb{E}\left[Y_{q}\right]+o\left(n^{q} a_{n}^{q / k}\right)
\end{array} \\
=\left(1+O\left(a_{n}^{\frac{q}{2 k}}\right)\right) q ! n^{q} \int_{0}^{1} \cdots \int_{0}^{1} \int_{0}^{\infty} \int_{0}^{x_{1}} \begin{array}{c}
x_{0-1} \\
G_{\widehat{V}_{n}}\left(\mathbf{t}_{q}, \mathbf{x}_{q}\right) e^{-\sum_{i=1}^{q} x_{i}} \mathrm{~d} \overleftarrow{\mathbf{x}}_{q} \mathrm{~d} \overleftarrow{\mathbf{t}}_{q}+ \\
+\mathbb{E}\left[Y_{q}\right]+o\left(n^{q} a_{n}^{q / k}\right) ;
\end{array}
\end{gathered}
$$

note that if we had not excluded the root, we would not be able to write the sum as an integral. By making the change of variables $x_{i}=a_{n}^{1 / k} w_{i}$, for $1 \leqslant i \leqslant q$, we have that

$$
\mathbb{E}\left[X_{q}\right]=\left(1+O\left(a_{n}^{\frac{q}{2 k}}\right)\right) q ! n^{q} a_{n}^{q / k} \int_{0}^{1} \cdots \int_{0}^{1} \bar{H}_{n, q}\left(\mathbf{t}_{q}\right) \mathrm{d} \overleftarrow{\mathbf{t}}_{q}+\mathbb{E}\left[Y_{q}\right]++o\left(n^{q} a_{n}^{q / k}\right) .
$$

Finally, our claim follows by induction on $q \in \mathbb{N}$ and the assumption $\lim _{n \rightarrow \infty} n a_{n}^{1 / k}=$ $\infty$.

We are now able to establish Lemma 3.

Proof of Lemma 3. First note that by condition (a) of Lemma 3 and (13), we have $\max _{v \in \mathbb{T}_{n}} d_{n}(v)=\sup _{t \in[0,1]} \widetilde{V}_{n}(t)=O\left(a_{n}^{-1}\right)$. Thus the conditions for Lemma 5 and Lemma 6 are satisfied.

Recall the functions $\bar{H}_{n, q}$ and $H_{f, q}$ defined in (15) and (10), respectively. Therefore, notice that we only need to show that

$$
\int_{[0,1]^{q}} \bar{H}_{n, q}\left(\mathbf{t}_{q}\right) \mathrm{d} \overleftarrow{\mathbf{t}}_{q} \rightarrow \int_{[0,1]^{q}} H_{f, q}\left(\mathbf{t}_{q}\right) \mathrm{d} \overleftarrow{\mathbf{t}}_{q}, \quad \text { as } n \rightarrow \infty
$$

The above convergence together with Lemma 6 implies that $\mathbb{E}\left[\mathcal{K}_{1}\left(\mathbb{T}_{n}\right)^{q}\right]=O\left(n^{q} a_{n}^{q / k}\right)$ which clearly proves the first claim in Lemma 3. The second claim follows immediately from Theorem 2 and the method of moments. 
We henceforth prove the claim in (18). Recall that a sequence $\left(g_{n}\right)_{n \geqslant 1}$ of non-negative functions on a measure space $(\Omega, \mathcal{F}, \mu)$ with total mass 1 , i.e., $\mu(\Omega)=1$, is uniformly integrable if $\int_{\Omega} g_{n} \mathrm{~d} \mu<\infty$ for all $n \geqslant 1$ and

$$
\sup _{A \in \mathcal{F}: \mu(A) \leqslant \delta} \sup _{n \geqslant 1} \int_{A} g_{n} \mathrm{~d} \mu \rightarrow 0, \quad \text { as } \delta \rightarrow 0 .
$$

We also recall the following useful result on uniformly integrable sequences of functions. Suppose further that $g_{n} \rightarrow g$ almost everywhere as $n \rightarrow \infty$. By [27, Proposition 4.12], we know that

$$
\left(g_{n}\right)_{n \geqslant 1} \text { is uniformly integrable if and only if } \int g_{n} \mathrm{~d} \mu \rightarrow \int g \mathrm{~d} \mu<\infty,
$$

as $n \rightarrow \infty$. Then in order to prove (18), it is enough to check the following:

(i) The sequence $\left(\bar{H}_{n, q}\right)_{n \geqslant 1}$ is uniformly integrable on $[0,1]^{q}$, and

(ii) $\bar{H}_{n, q} \rightarrow H_{f, q}$ as $n \rightarrow \infty$.

We start by showing (i). Note that $\left|a_{n} \widetilde{V}_{n}(t)-a_{n} \widehat{V}_{n}(t)\right| \leqslant a_{n}$ for $t \in[0,1]$. Then, the assumption (a) implies that $a_{n} \widehat{V}_{n}(t) \rightarrow f(t)$ and $1 /\left(a_{n} \widehat{V}_{n}(t)\right)^{1 / k} \rightarrow(1 / f(t))^{1 / k}$, for every $t \in[0,1]$, as $n \rightarrow \infty$. Moreover, the assumption (b) shows that $\left(1 /\left(a_{n} \widehat{V}_{n}(t)\right)^{1 / k}\right)_{n \geqslant 1}$ is uniformly integrable on $[0,1]$. More generally, for every fixed $q \in \mathbb{N}$ and $\mathbf{t}_{q}=\left(t_{1}, \ldots, t_{q}\right)$, define the function $\widetilde{H}_{n, q}\left(\mathbf{t}_{q}\right):=\left(a_{n} \widehat{V}_{n}\left(t_{1}\right) \cdots a_{n} \widehat{V}_{n}\left(t_{q}\right)\right)^{-1 / k}$. We then observe that

$$
\begin{aligned}
\int_{0}^{1} \cdots \int_{0}^{1} \widetilde{H}_{n, q}\left(\mathbf{t}_{q}\right) \mathrm{d} \mathbf{t}_{q} & =\left(\int_{0}^{1}\left(a_{n} \widehat{V}_{n}(t)\right)^{-1 / k} \mathrm{~d} t\right)^{q} \\
& \rightarrow\left(\int_{0}^{1} f(t)^{-1 / k} \mathrm{~d} t\right)^{q} \\
& =\int_{0}^{1} \cdots \int_{0}^{1}\left(f\left(t_{1}\right) \cdots f\left(t_{q}\right)\right)^{-1 / q} \mathrm{~d} \mathbf{t}_{q},
\end{aligned}
$$

as $n \rightarrow \infty$. Thus the result in (19) shows that the sequence $\left(\widetilde{H}_{n, q}\right)_{n \geqslant 1}$ is uniformly integrable on $[0,1]^{q}$. Next notice that the inequality $\exp \left(-a_{n}^{1 / k}\left(x_{1}+\cdots+x_{q}\right)\right) \leqslant 1$ implies that $\bar{H}_{n, q}\left(\mathbf{t}_{q}\right) \leqslant H_{a_{n} \widehat{V}_{n}, q}\left(\mathbf{t}_{q}\right)$, where $H_{a_{n} \widehat{V}_{n}, q}$ is defined in (10). Then the inequality (11) implies that there exists a constant $C_{k, q}>0$ such that $\bar{H}_{n, q}\left(\mathbf{t}_{q}\right) \leqslant C_{k, q} \widetilde{H}_{n, q}\left(\mathbf{t}_{q}\right)$. Hence (i) follows by applying [18, Theorem 4.5].

Finally, we verify (ii). Recall that condition (a) implies that $a_{n} \widehat{V}_{n}(t) \rightarrow f(t)$, for every $t \in[0,1]$, as $n \rightarrow \infty$. Hence, whenever $0 \leqslant t_{1} \leqslant t_{2} \leqslant 1, \inf _{t \in\left[t_{1}, t_{2}\right]} a_{n} \widehat{V}_{n}(t) \rightarrow \inf _{t \in\left[t_{1}, t_{2}\right]} f(t)$ as $n \rightarrow \infty$. Thus, for $q \in \mathbb{N}$, the equation (7), implies that

$$
D_{a_{n} \widehat{V}_{n}}\left(t_{1}, \ldots, t_{q}\right) \rightarrow D_{f}\left(t_{1}, \ldots, t_{q}\right)
$$


uniformly for $t_{1}, \ldots, t_{q} \in[0,1]$ as $n \rightarrow \infty$. Then, for $\mathbf{x}_{q} \in \mathbb{R}_{+}^{q}$ and $\mathbf{t}_{q} \in[0,1]^{q}$,

$$
G_{a_{n} \widehat{V}_{n}}\left(\mathbf{t}_{q}, \mathbf{x}_{q}\right) e^{-a_{n}^{1 / k} \sum_{i=1}^{q} x_{i}} \rightarrow G_{f}\left(\mathbf{t}_{q}, \mathbf{x}_{q}\right) \text {, as } n \rightarrow \infty .
$$

Note that for $\varepsilon \in(0,1)$ there exists $N \in \mathbb{N}$ such that

$$
G_{a_{n} \widehat{V}_{n}}\left(\mathbf{t}_{q}, \mathbf{x}_{q}\right) e^{-a_{n}^{1 / k} \sum_{i=1}^{q} x_{i}} \leqslant \exp \left(-\frac{(1-\varepsilon) f\left(t_{1}\right) x_{1}^{1 / k}}{k !}\right), \text { for } n \geqslant N .
$$

Moreover, note that condition (b) implies that the function on the right-hand side of the inequality is integrable on $\left\{\mathbf{x}_{q} \in \mathbb{R}_{+}: 0 \leqslant x_{q} \leqslant \cdots \leqslant x_{1}<\infty\right\}$. Therefore, it should be clear that (ii) follows by the dominated convergence theorem. This finishes the proof.

We can apply similar ideas as in the proofs of Lemma 3 and Lemma 6 to estimate the mean of the number of $r$-records $\mathcal{K}_{r}\left(\mathbb{T}_{n}\right)$. It is important to mention that we have not tried to estimate higher moments of $\mathcal{K}_{r}\left(\mathbb{T}_{n}\right)$ to obtain a limit theorem in distribution for this quantity. We believe that our methods can be used but the computations will be more involved and we decided not to do it. Furthermore, the next results show that $\mathcal{K}_{r}\left(\mathbb{T}_{n}\right)$ is of smaller order than $\mathcal{K}_{1}\left(\mathbb{T}_{n}\right)$ and hence it will not contribute (in the limit) to the distribution of the $k$-cut number $\mathcal{K}\left(\mathbb{T}_{n}\right)$.

Lemma 7. Let $\mathbb{T}_{n}$ be an ordered (deterministic) rooted tree with $n \in \mathbb{N}$ vertices. Suppose that there exists a sequence $\left(a_{n}\right)_{n \geqslant 1}$ of non-negative real numbers with $\lim _{n \rightarrow \infty} a_{n}=0$, $\lim _{n \rightarrow \infty} n a_{n}^{1 / k}=\infty$ and $\max _{v \in \mathbb{T}_{n}} d_{n}(v)=O\left(a_{n}^{-1}\right)$. Then, for $r \in\{1, \ldots, k\}$,

$$
n^{-1} a_{n}^{-r / k} \mathbb{E}\left[\mathcal{K}_{r}\left(\mathbb{T}_{n}\right)\right]=\left(1+O\left(a_{n}^{\frac{1}{2 k}}\right)\right) \int_{0}^{1} \int_{0}^{\infty} \frac{x^{r-1} e^{-a_{n}^{1 / k} x}}{\Gamma(r)} e^{-\frac{a_{n} \widehat{V}_{n}(t) x^{k}}{k !}} \mathrm{d} x \mathrm{~d} t+o(1) .
$$

Proof. Note that the case $r=1$ has been proven in Lemma 6. We follow a similar strategy to prove the case $r \in\{2, \ldots, k\}$. Recall that $I_{r, v}$ is the indicator of the event that the vertex $v \in \mathbb{T}_{n}$ is an $r$-record defined in (4). We observe that

$$
\begin{aligned}
\mathbb{E}\left[I_{r, v}\right] & =\int_{0}^{\infty} \frac{x^{r-1} e^{-x}}{\Gamma(r)} \mathbb{P}(\operatorname{Gamma}(\mathrm{k})>x)^{d_{n}(v)} \mathrm{d} x \\
& =\int_{0}^{x_{0}} \frac{x^{r-1} e^{-x}}{\Gamma(r)} \mathbb{P}(\operatorname{Gamma}(\mathrm{k})>x)^{d_{n}(v)} \mathrm{d} x+\int_{x_{0}}^{\infty} \frac{x^{r-1} e^{-x}}{\Gamma(r)} \mathbb{P}(\operatorname{Gamma}(\mathrm{k})>x)^{d_{n}(v)} \mathrm{d} x \\
& =A_{1}+A_{2},
\end{aligned}
$$

where $x_{0}^{\alpha}=a_{n}^{\alpha}$ and $\alpha=\frac{1}{2}\left(\frac{1}{k}+\frac{1}{k+1}\right)$. On the one hand, Lemma 5, with $q=1$, implies that

$$
A_{2} \leqslant O\left(e^{-\frac{x_{0}^{k}}{2 a_{n} k !}}\right) \int_{0}^{\infty} x^{r-1} e^{-x} \mathrm{~d} x=O\left(e^{-\frac{x_{0}^{k}}{2 a_{n} k !}}\right) .
$$

On the other hand, Lemma 5 , with $q=1$, also implies that

$$
A_{1}=\left(1+O\left(a_{n}^{\frac{1}{2 k}}\right)\right) \int_{0}^{x_{0}} \frac{x^{r-1} e^{-x}}{\Gamma(r)} e^{-\frac{d_{n}(v) x^{k}}{k !}} \mathrm{d} x
$$




$$
=\left(1+O\left(a_{n}^{\frac{1}{2 k}}\right)\right) \int_{0}^{\infty} \frac{x^{r-1} e^{-x}}{\Gamma(r)} e^{-\frac{d_{n}(v) x^{k}}{k !}} \mathrm{d} x+A_{3}
$$

where

$$
A_{3}=\int_{x_{0}}^{\infty} \frac{x^{r-1} e^{-x}}{\Gamma(r)} e^{-\frac{d_{n}(v) x^{k}}{k !}} \mathrm{d} x=O\left(e^{-\frac{x_{0}^{k}}{2 a_{n} k !}}\right)
$$

this estimate can be deduced similarly as the one for the integral $A_{2}$. By recalling that $\mathcal{K}_{r}\left(\mathbb{T}_{n}\right)=\sum_{v \in \mathbb{T}_{n}} I_{r, v}$, we conclude from the previous estimations that

$$
\begin{aligned}
\mathbb{E}\left[\mathcal{K}_{r}\left(\mathbb{T}_{n}\right)\right] & =\left(1+O\left(a_{n}^{\frac{1}{2 k}}\right)\right) \sum_{v \in \mathbb{T}_{n} \backslash\{0\}} \int_{0}^{\infty} \frac{x^{r-1} e^{-x}}{\Gamma(r)} e^{-\frac{d_{n}(v) x^{k}}{k !}} \mathrm{d} x+o\left(n a_{n}^{r / k}\right) \\
& =\left(1+O\left(a_{n}^{\frac{1}{2 k}}\right)\right) 2^{-1} \int_{0}^{2(n-1)} \int_{0}^{\infty} \frac{x^{r-1} e^{-x}}{\Gamma(r)} e^{-\frac{\left\lceil V_{n}(t)\right] x^{k}}{k !}} \mathrm{d} x \mathrm{~d} t+o\left(n a_{n}^{r / k}\right) \\
& =\left(1+O\left(a_{n}^{\frac{1}{2 k}}\right)\right) n \int_{0}^{1} \int_{0}^{\infty} \frac{x^{r-1} e^{-x}}{\Gamma(r)} e^{-\frac{\widehat{V}_{n}(t) x^{k}}{k !}} \mathrm{d} x \mathrm{~d} t+o\left(n a_{n}^{r / k}\right) .
\end{aligned}
$$

Finally, our claim follows by making the change of variables $x=a_{n}^{1 / k} w$.

Lemma 8. Suppose that $\left(\mathbb{T}_{n}\right)_{n \geqslant 1}$ is a sequence of ordered (deterministic) rooted trees. Suppose that there exists a sequence $\left(a_{n}\right)_{n \geqslant 1}$ of non-negative real numbers with $\lim _{n \rightarrow \infty} a_{n}=0$, $\lim _{n \rightarrow \infty} n a_{n}^{1 / k}=\infty$, and a function $f \in C\left([0,1], \mathbb{R}_{+}\right)$such that $\widetilde{V}_{n}$ satisfies the condition (a) in Lemma 3 and that for $r \in\{1, \ldots, k\}$,

$$
\int_{0}^{1}\left(a_{n} \widehat{V}_{n}(t)\right)^{-r / k} \mathrm{~d} t \rightarrow \int_{0}^{1} f(t)^{-r / k} \mathrm{~d} t<\infty, \quad \text { as } n \rightarrow \infty .
$$

Then,

$$
n^{-1} a_{n}^{-r / k} \mathbb{E}\left[\mathcal{K}_{r}\left(\mathbb{T}_{n}\right)\right] \rightarrow \frac{(k !)^{r / k} \Gamma(r / k)}{k \Gamma(r)} \int_{0}^{1} f(t)^{-r / k} \mathrm{~d} t, \text { as } n \rightarrow \infty .
$$

Proof. Notice that the case $r=1$ has been proved in Lemma 3. The proof of the general case $r \in\{1, \ldots, k\}$ follows by a simple adaptation of the argument used in the proof of Lemma 3 for $q=1$ with the use of Lemma 7 . One only needs to note that

$$
\int_{0}^{1} \int_{0}^{\infty} \frac{x^{r-1}}{\Gamma(r)} e^{-\frac{f(t) x^{k}}{k !}} \mathrm{d} x \mathrm{~d} t=\frac{(k !)^{\frac{r}{k}}}{k} \frac{\Gamma\left(\frac{r}{k}\right)}{\Gamma(r)} \int_{0}^{1} f(t)^{-r / k} \mathrm{~d} t .
$$

\section{Proof of Theorem 1}

Let $\mathbb{T}_{n}$ be a Galton-Watson tree conditioned on its number of vertices being $n \in \mathbb{N}$ with offspring distribution $\xi$ satisfying (1). Note that in this case both the $r$-records and the tree are random. Then we study $\mathcal{K}_{r}\left(\mathbb{T}_{n}\right)$ as a random variable conditioned on $\mathbb{T}_{n}$. More precisely, we first choose a random tree $\mathbb{T}_{n}$. Then we keep it fixed and consider the number of $r$-records. This gives a random variable $\mathcal{K}_{r}\left(\mathbb{T}_{n}\right)$ with distribution that depends on $\mathbb{T}_{n}$. We have the following lemma that corresponds to [25, Lemma 4.8]. 
Lemma 9. Let $\mathbb{T}_{n}$ be a Galton-Watson tree conditioned on its number of vertices being $n \in \mathbb{N}$ with offspring distribution $\xi$ satisfying (1). For $r \in\{1, \ldots, k\}$. We have that $\mathbb{E}\left[\mathcal{K}_{r}\left(\mathbb{T}_{n}\right)\right]=O\left(n^{1-\frac{r}{2 k}}\right)$.

Proof. By an application of the proof of Lemma 7 with $a_{n}=n^{-1 / 2}$ (in particular, the equality (20)), we see that

$$
\begin{aligned}
\mathbb{E}\left[\mathcal{K}_{r}\left(\mathbb{T}_{n}\right) \mid \mathbb{T}_{n}\right] & \leqslant\left(1+O\left(a_{n}^{\frac{1}{2 k}}\right)\right) \sum_{v \in \mathbb{T}_{n} \backslash\{\circ\}} \int_{0}^{\infty} \frac{x^{r-1}}{\Gamma(r)} e^{-\frac{d_{n}(v) x^{k}}{k !}} \mathrm{d} x+o\left(n a_{n}^{r / k}\right) \\
& =\left(1+O\left(a_{n}^{\frac{1}{2 k}}\right)\right) \sum_{v \in \mathbb{T}_{n} \backslash\{\circ\}} \frac{(k !)^{r / k} \Gamma(r / k)}{k \Gamma(r)} d_{n}(v)^{-r / k}+o\left(n a_{n}^{r / k}\right) \\
& =\left(1+O\left(a_{n}^{\frac{1}{2 k}}\right)\right) \frac{(k !)^{r / k} \Gamma(r / k)}{k \Gamma(r)} \sum_{i=1}^{\infty} i^{-r / k} w_{i}\left(\mathbb{T}_{n}\right)+o\left(n a_{n}^{r / k}\right),
\end{aligned}
$$

where $w_{i}\left(\mathbb{T}_{n}\right)$ denotes the number of vertices at depth $i \in \mathbb{N}$ in $\mathbb{T}_{n}$. Notice that

$$
\sum_{i=1}^{\infty} i^{-r / k} w_{i}\left(\mathbb{T}_{n}\right) \leqslant n^{1-\frac{r}{2 k}}+\sum_{i=1}^{\left\lfloor n^{1 / 2}\right\rfloor} i^{-r / k} w_{i}\left(\mathbb{T}_{n}\right)
$$

by the fact that $\sum_{i \geqslant 0} w_{i}\left(\mathbb{T}_{n}\right)=n$. Since $\mathbb{E}\left[\xi^{2}\right]<\infty$ by our assumption (1), [25, Theorem 1.13] implies that for all $n, i \in \mathbb{N}, \mathbb{E}\left[w_{i}\left(\mathbb{T}_{n}\right)\right] \leqslant C i$ for some constant $C>0$ depending on $\xi$ only. Therefore,

$$
\sum_{i=1}^{\infty} i^{-r / k} \mathbb{E}\left[w_{i}\left(\mathbb{T}_{n}\right)\right] \leqslant n^{1-\frac{r}{2 k}}+\sum_{i=1}^{\left\lfloor n^{1 / 2}\right\rfloor} \mathbb{E}\left[w_{i}\left(\mathbb{T}_{n}\right)\right] i^{-\frac{r}{k}}=O\left(n^{1-\frac{r}{2 k}}\right)
$$

By taking expectation in (21), our claim follows by (22).

We continue by studying the moments of the number of 1 -records $\mathcal{K}_{1}\left(\mathbb{T}_{n}\right)$. We denote by $\mu_{n}$ the (random) probability distribution of $\sigma^{-1 / k} n^{-1+1 / 2 k} \mathcal{K}_{1}\left(\mathbb{T}_{n}\right)$ given $\mathbb{T}_{n}$. Define the random variables

$$
m_{q}\left(\mathbb{T}_{n}\right):=\mathbb{E}\left[\mathcal{K}_{1}\left(\mathbb{T}_{n}\right)^{q} \mid \mathbb{T}_{n}\right], \quad q \in \mathbb{Z}_{\geqslant 0}
$$

Notice that the moments of $\mu_{n}$ are given by $\sigma^{-q / k} n^{-q+q / 2 k} m_{q}\left(\mathbb{T}_{n}\right)$. We have the following lemma that corresponds to [25, Lemma 4.9].

Lemma 10. Let $\mathbb{T}_{n}$ be a Galton-Watson tree conditioned on its number of vertices being $n \in \mathbb{N}$ with offspring distribution $\xi$ satisfying (1). Furthermore, suppose that for every fixed $q \in \mathbb{N}$ we have that $\mathbb{E}\left[\xi^{q+1}\right]<\infty$. Then $\mathbb{E}\left[m_{q}\left(\mathbb{T}_{n}\right)\right]=O\left(n^{q-\frac{q}{2 k}}\right)$. 
Proof. By an application of Lemma 6 with $q \in \mathbb{N}$ and $a_{n}=n^{-1 / 2}$ (in particular, the equality (17) in its proof), we see that

$$
m_{q}\left(\mathbb{T}_{n}\right) \leqslant\left(1+O\left(n^{-\frac{q}{4 k}}\right)\right) q ! \sum_{v_{1}, \ldots, v_{q} \in \mathbb{T}_{n} \backslash\{\circ\}} \int_{0}^{\infty} \int_{0}^{x_{1}} \cdots \int_{0}^{x_{q-1}} G_{n}\left(\mathbf{v}_{q}, \mathbf{x}_{q}\right) \mathrm{d} \overleftarrow{\mathbf{x}}_{q}+Y_{q},
$$

where $Y_{q}:=\sum_{p=0}^{q-1} \sum_{l=0}^{p}\left(\begin{array}{l}q \\ p\end{array}\right)\left(\begin{array}{l}p \\ l\end{array}\right)(-1)^{p-l} m_{l}\left(\mathbb{T}_{n}\right)$. After a similar computation as in the proof of the inequality (11), one sees that there exists a constant $C_{k, q}>0$ such that

$$
m_{q}\left(\mathbb{T}_{n}\right) \leqslant\left(1+O\left(n^{-\frac{q}{4 k}}\right)\right) q ! C_{k, q} \bar{m}_{1}\left(\mathbb{T}_{n}\right)^{q}+Y_{q},
$$

where $\bar{m}_{1}\left(\mathbb{T}_{n}\right):=\sum_{v \in \mathbb{T}_{n} \backslash\{0\}} d_{n}(v)^{-1 / k}$. Notice that

$$
\bar{m}_{1}\left(\mathbb{T}_{n}\right)=\sum_{i=1}^{\infty} w_{i}\left(\mathbb{T}_{n}\right) i^{-\frac{1}{k}} \leqslant n^{1-\frac{1}{2 k}}+\sum_{i=1}^{\left\lfloor n^{1 / 2}\right\rfloor} w_{i}\left(\mathbb{T}_{n}\right) i^{-\frac{1}{k}},
$$

where $w_{i}\left(\mathbb{T}_{n}\right)$ denotes the number of vertices at depth $i \in \mathbb{N}$ in $\mathbb{T}_{n}$. Since $\mathbb{E}\left[\xi^{q+1}\right]<\infty$ for $q \in \mathbb{N}$, [25, Theorem 1.13] implies that for all $n, i \in \mathbb{N}, \mathbb{E}\left[w_{i}\left(\mathbb{T}_{n}\right)^{q}\right] \leqslant C i^{q}$ for some constant $C>0$ depending on $q$ and $\xi$ only. Therefore, Minkowski's inequality implies that

$$
\mathbb{E}\left[\bar{m}_{1}\left(\mathbb{T}_{n}\right)^{q}\right]^{\frac{1}{q}} \leqslant n^{1-\frac{1}{2 k}}+\sum_{i=1}^{\left\lfloor n^{1 / 2}\right\rfloor} \mathbb{E}\left[w_{i}\left(\mathbb{T}_{n}\right)^{q}\right]^{\frac{1}{q}} i^{-\frac{1}{k}}=O\left(n^{1-\frac{1}{2 k}}\right) .
$$

By taking expectation in (23), we deduce from (24) that

$$
\mathbb{E}\left[m_{q}\left(\mathbb{T}_{n}\right)\right]=\mathbb{E}\left[Y_{q}\right]+O\left(n^{q-\frac{q}{2 k}}\right),
$$

and our claim follows by induction on $q \in \mathbb{N}$.

Let $\widetilde{V}_{n}$ and $\widehat{V}_{n}$ be the normalized depth-first search walks associated with the conditioned Galton-Watson tree $\mathbb{T}_{n}$. Note that in this case $\widetilde{V}_{n}$ becomes a random function on $C\left([0,1], \mathbb{R}_{+}\right)$. Recall that a remarkable result due to Aldous [3, Theorem 23 with Remark 2] (see also [29, Theorem 1]) shows that

$$
n^{-1 / 2} \widetilde{V}_{n} \stackrel{d}{\rightarrow} 2 \sigma^{-1} B^{\mathrm{ex}}, \quad \text { as } n \rightarrow \infty,
$$

in $C\left([0,1], \mathbb{R}_{+}\right)$, with its usual topology, and where $B^{\mathrm{ex}}=\left(B^{\mathrm{ex}}(t), t \in[0,1]\right)$ is a standard normalized Brownian excursion. Note that $B^{\text {ex }}$ is a random element from $C\left([0,1], \mathbb{R}_{+}\right)$; see for example [8] or [36].

Lemma 11. For $r \in\{1, \ldots, k\}$, we have that $\int_{0}^{1} B^{\operatorname{ex}}(t)^{-r / k} \mathrm{~d} t<\infty$ almost surely.

Proof. One only needs to show that $\mathbb{E}\left[\int_{0}^{1} B^{\operatorname{ex}}(t)^{-r / k} \mathrm{~d} t\right]<\infty$. This follows by computing $\mathbb{E}\left[B^{\operatorname{ex}}(t)^{-r / k}\right]$, for every $t \in[0,1]$, from the well-known density function of $B^{\text {ex }}(t)$; see $[8$, Chapter II, Equation (1.4)]. 
Therefore, Theorem 2 and Lemma 11 imply that there exists almost surely a (unique) measure $\nu_{2} B_{\text {ex }}$ with moments given by $m_{q}\left(2 B^{\text {ex }}\right)$. The next result provides a generalization of [25, Theorem 1.10] and it will be used in the proof of Theorem 1.

Theorem 12. Let $\mathbb{T}_{n}$ be a Galton-Watson tree conditioned on its number of vertices being $n \in \mathbb{N}$ with offspring distribution $\xi$ satisfying (1). Then

$$
\mu_{n} \stackrel{d}{\rightarrow} \nu_{2 B^{\mathrm{ex}}}, \quad \text { as } n \rightarrow \infty
$$

in the space of probability measures on $\mathbb{R}$. Moreover, we have that for every $q \in \mathbb{N}$,

$$
\sigma^{-q / k} n^{-q+q / 2 k} m_{q}\left(\mathbb{T}_{n}\right) \stackrel{d}{\rightarrow} m_{q}\left(2 B^{\mathrm{ex}}\right), \quad \text { as } n \rightarrow \infty .
$$

The convergences in (25), (26) and (27), for all $q \in \mathbb{N}$, hold jointly. In particular, if $\mathbb{E}\left[\xi^{p}\right]<\infty$ for all $p \in \mathbb{N}$, then for all $q \in \mathbb{N}$ and $l \in \mathbb{N}$,

$$
\sigma^{-l q / k} n^{-l q / k+l q / 2 k} \mathbb{E}\left[m_{q}\left(\mathbb{T}_{n}\right)^{l}\right] \rightarrow \mathbb{E}\left[m_{q}\left(2 B^{\mathrm{ex}}\right)^{l}\right], \quad \text { as } n \rightarrow \infty .
$$

Proof. A simple adaptation of the proof of [25, Lemma 4.7] easily shows that

$$
\left(\widetilde{V}_{n}, \int_{0}^{1} \widehat{V}_{n}(t)^{-1 / k} \mathrm{~d} t\right) \stackrel{d}{\rightarrow}\left(2 \sigma^{-1} B^{\mathrm{ex}}, 2^{-1 / k} \sigma^{1 / k} \int_{0}^{1} B^{\mathrm{ex}}(t)^{-1 / k} \mathrm{~d} t\right)
$$

in $C\left([0,1], \mathbb{R}_{+}\right) \times \mathbb{R}$, as $n \rightarrow \infty$. By the Skorohod coupling theorem (see e.g. [27, Theorem $4.30])$, we can assume that the trees $\left(\mathbb{T}_{n}\right)_{n \geqslant 1}$ are defined on a common probability space such that the convergence in (29) holds almost surely. Therefore, the convergences (26) and (27) follow immediately from Lemma 3. It only remains to prove (28). Recall that we assume that $\mathbb{E}\left[\xi^{p}\right]<\infty$ for every $p \in \mathbb{N}$. By Jensen's inequality, we notice that $m_{q}\left(\mathbb{T}_{n}\right)^{l} \leqslant m_{l q}\left(\mathbb{T}_{n}\right)$ for $l, q \in \mathbb{N}$. Hence Lemma 10 implies that $\mathbb{E}\left[m_{q}\left(\mathbb{T}_{n}\right)^{l}\right]=O\left(n^{l q-\frac{l q}{2 k}}\right)$. This shows that every moment of the right-hand side of (27) stays bounded as $n \rightarrow \infty$ which implies (28).

We are now able to prove Theorem 1.

Proof of Theorem 1. Lemma 9 establishes that $\mathbb{E}\left[\mathcal{K}_{r}\left(\mathbb{T}_{n}\right)\right]=O\left(n^{1-\frac{r}{2 k}}\right)$ for $r \in\{1, \ldots, k\}$. As a consequence, Markov's inequality implies that $n^{-1+\frac{1}{2 k}} \mathcal{K}_{r}\left(\mathbb{T}_{n}\right) \rightarrow 0$ in probability, as $n \rightarrow \infty$, for $r \in\{2, \ldots, k\}$. Then, by the identity in (5), it is enough to prove Theorem 1 for $\mathcal{K}_{1}\left(\mathbb{T}_{n}\right)$ instead of $\mathcal{K}\left(\mathbb{T}_{n}\right)$. By the definition of $\mu_{n}$ and Theorem 12 , for any bounded continuous function $g: \mathbb{R}_{+} \rightarrow \mathbb{R}_{+}$,

$$
\mathbb{E}\left[g\left(\sigma^{-1 / k} n^{-1+1 / 2 k} \mathcal{K}_{1}\left(\mathbb{T}_{n}\right)\right) \mid \mathbb{T}_{n}\right]=\int g \mathrm{~d} \mu_{n} \stackrel{d}{\rightarrow} \int g \mathrm{~d} \nu_{2 B^{\text {ex }}}, \quad \text { as } n \rightarrow \infty .
$$

Taking expectations, the dominated convergence theorem implies that

$$
\sigma^{-1 / k} n^{-1+1 / 2 k} \mathcal{K}_{1}\left(\mathbb{T}_{n}\right) \stackrel{d}{\rightarrow} Z_{\mathrm{CRT}}, \quad \text { as } n \rightarrow \infty,
$$


where $Z_{\mathrm{CRT}}$ has distribution $\nu(\cdot)=\mathbb{E}\left[\nu_{2 B^{\operatorname{ex}}}(\cdot)\right]$. Suppose that $\mathbb{E}\left[\xi^{p}\right]<\infty$ for every $p \in \mathbb{N}$. Lemma 10 implies that every moment of $n^{-1+1 / 2 k} \mathcal{K}_{1}\left(\mathbb{T}_{n}\right)$ stays bounded as $n \rightarrow \infty$ which implies the moment convergence in Theorem 1 . It remains to identify the moments of $Z_{\mathrm{CRT}}$ (or equivalently $\nu$ ). Notice that

$$
\mathbb{E}\left[Z_{\mathrm{CRT}}^{q}\right]=\int x^{q} \mathrm{~d} \nu=\mathbb{E}\left[\int x^{q} \mathrm{~d} \nu_{2 B^{\text {ex }}}\right]=\mathbb{E}\left[m_{q}\left(2 B^{\text {ex }}\right)\right], \quad \text { for } q \in \mathbb{N} .
$$

For $q \in \mathbb{N}$, let $U_{1}, \ldots, U_{q}$ be independent random variables with the uniform distribution on $[0,1]$. Let $Y_{1}, \ldots, Y_{q}$ be the first $q$ points in a Poisson process on $(0, \infty)$ with intensity $x \mathrm{~d} x$, i.e., $Y_{1}, \ldots, Y_{q}$ have joint density function $y_{1} \cdots y_{q} e^{-y_{q}^{2} / 2}$ on $0<y_{1}<\cdots<y_{q}<\infty$. It is well-known that $L_{2 B^{\text {ex }}}\left(U_{1}, \ldots, U_{q}\right) \stackrel{d}{=} Y_{q}$, see, e.g., [25, Proof of Lemma 5.1]. Thus by recalling the definition of the function $H_{2 B^{\mathrm{ex}}, q}$ in (10), we see that

$$
\mathbb{E}\left[m_{q}\left(2 B^{\mathrm{ex}}\right)\right]=q ! \mathbb{E}\left[H_{2 B^{\mathrm{ex}}, q}\left(\mathbf{U}_{q}\right)\right]=q ! \int_{0}^{y_{1}} \cdots \int_{0}^{y_{q-1}} \int_{0}^{\infty} y_{1} \cdots y_{q} e^{-y_{q}^{2} / 2} \widetilde{F}_{q}\left(\mathbf{y}_{q}\right) \mathrm{d} \mathbf{y}_{q},
$$

where $\mathbf{U}_{q}=\left(U_{1}, \ldots, U_{q}\right), \mathbf{y}_{q}=\left(y_{1}, \ldots, y_{q}\right) \in \mathbb{R}_{+}^{q}$ and

$$
\widetilde{F}_{q}\left(\mathbf{y}_{q}\right):=\int_{0}^{\infty} \int_{0}^{x_{1}} \cdots \int_{0}^{x_{q-1}} \exp \left(-\frac{y_{1} x_{1}^{k}+\left(y_{2}-y_{1}\right) x_{1}^{k}+\cdots+\left(y_{q}-y_{q-1}\right) x_{q}^{k}}{k !}\right) \mathrm{d} \overleftarrow{\mathbf{x}}_{q} .
$$

Finally, the expression for the moments in Theorem 1 follows by first changing the order of integration in (30) and then by making the change of variables $w_{i}=y_{i}-y_{i-1}$ for $2 \leqslant i \leqslant q$.

Following the idea of the proof of Theorem 1, we obtain the following convergence of the first moment of the number of $r$-records $\mathcal{K}_{r}\left(\mathbb{T}_{n}\right)$. This provides a proof of [12, Lemma 4.10].

Lemma 13. Let $\mathbb{T}_{n}$ be a Galton-Watson tree conditioned on its number of vertices being $n \in \mathbb{N}$ with offspring distribution $\xi$ satisfying (1). For $r \in\{1, \ldots k\}$, we have that

$$
n^{-1+\frac{r}{2 k}} \mathbb{E}\left[\mathcal{K}_{r}\left(\mathbb{T}_{n}\right)\right] \rightarrow \frac{(k !) \frac{r}{k}}{k} \frac{\Gamma\left(\frac{r}{k}\right) \Gamma\left(1-\frac{r}{2 k}\right)}{\Gamma(r)}\left(\frac{\sigma}{\sqrt{2}}\right)^{\frac{r}{k}}, \text { as } n \rightarrow \infty .
$$

Proof. The proof follows by a simple adaptation of the argument used in the proof of Theorem 1 by using Lemma 8 (with $a_{n}=n^{-1 / 2}$ ), Lemma 9 and Lemma 11. One only needs to note that

$$
\mathbb{E}\left[\int_{0}^{1} B^{\mathrm{ex}}(t)^{-r / k} \mathrm{~d} t\right]=2^{-\frac{r}{2 k}} \Gamma\left(1-\frac{r}{2 k}\right)
$$

which follows from the well-known density function of $B^{\operatorname{ex}}(t)$; see [8, Chapter II, Equation $(1.4)]$. 


\section{Further applications}

In this section, we show that the results obtained in Section 2 can be used and extended to study the $k$-cut model in other families of trees. In this section, let $\mathbb{T}_{n}$ be a rooted tree (maybe random and not necessarily ordered) with $n \in \mathbb{N}$ vertices and root $\circ$.

\subsection{Paths}

Lemma 14. Let $\mathbb{T}_{n}$ be a path with $n$ vertices labelled $1, \ldots, n$ from the root to the leaf. For $k \in\{2,3, \ldots\}$, we have that $n^{-1+1 / k} \mathcal{K}\left(\mathbb{T}_{n}\right) \stackrel{d}{\rightarrow} Z_{\text {path }}$, as $n \rightarrow \infty$, where $Z_{\text {path }}$ is a nondegenerate random variable whose law is determined entirely by its moments: $\mathbb{E}\left[Z_{\text {path }}^{q}\right]=$ $m_{q}(f)$ for $q \in \mathbb{Z}_{\geqslant 0}$, where

$$
f(t)= \begin{cases}2 t, & t \in[0,1 / 2] \\ 2-2 t, & t \in(1 / 2,1]\end{cases}
$$

Proof. By [12, Theorem 1.1], we know that $\mathbb{E}\left[\mathcal{K}_{r}\left(\mathbb{T}_{n}\right)\right]=O\left(n^{1-\frac{r}{k}}\right)$, for $r \in\{1, \ldots, k-1\}$, and $\mathbb{E}\left[\mathcal{K}_{k}\left(\mathbb{T}_{n}\right)\right]=O(\ln n)$. Then Markov's inequality implies that $n^{-1+1 / k} \mathcal{K}_{r}\left(\mathbb{T}_{n}\right) \rightarrow 0$ in probability, as $n \rightarrow \infty$, for $r \in\{2, \ldots, k\}$. Thus, by the identity (5), it is enough to prove our result for $\mathcal{K}_{1}\left(\mathbb{T}_{n}\right)$ instead of $\mathcal{K}\left(\mathbb{T}_{n}\right)$. Note that the normalized depth-first search walks $\widetilde{V}_{n}$ and $\widehat{V}_{n}$ of $\mathbb{T}_{n}$, defined in (12), are given by $n^{-1} \widetilde{V}_{n}(t)=f(t)$. and that $n^{-1} \widehat{V}_{n}(t)=n^{-1}\left\lceil\widetilde{V}_{n}(t)\right\rceil$ for $t \in[0,1]$. It should be clear that the conditions of Lemma 3 are fulfilled with $a_{n}=n^{-1}$. Therefore, our result follows from a simple application of Lemma 3.

Remark 15 . The convergence in distribution and moments of the $k$-cut number of a path to $Z_{\text {path }}$ has been proved in [12, Theorem 1.5] with a very different method. The contribution of Lemma 14 is the formula for computing the $q$-th moment of the limiting variable $Z_{\text {path }}$ for all $q \in \mathbb{Z}_{\geqslant 0}$.

\subsection{General trees}

The next result establishes a limit in distribution for the number of 1-records $\mathcal{K}_{1}\left(\mathbb{T}_{n}\right)$ of a general (random) rooted tree in the same spirit as in Lemma 3. For $q \in \mathbb{N}$, let $u_{1}, \ldots, u_{q}$ be a sequence of independent uniformly chosen vertices on $\mathbb{T}_{n}$. Recall that $L_{n}\left(u_{1}, \ldots, u_{q}\right)$ denotes the number of edges in the subtree of $\mathbb{T}_{n}$ spanned by $u_{1}, \ldots, u_{q}$ and its root o (i.e., the minimal number of edges that are needed to connect $u_{1}, \ldots, u_{q}$ and $\left.\circ\right)$. In particular, $L_{n}\left(u_{1}\right)=d_{n}\left(u_{1}\right)$ is the depth of the vertex $u_{1}$ in $\mathbb{T}_{n}$. In the sequel, we will often use the notation $A_{n}=O_{p}\left(B_{n}\right)$, where $\left(A_{n}\right)_{n \geqslant 1}$ and $\left(B_{n}\right)_{n \geqslant 1}$ are two sequences of non-negative real random variables such that $B_{n}>0$, to indicate that $\lim _{\delta \rightarrow \infty} \lim _{\sup _{n \rightarrow \infty}} \mathbb{P}\left(A_{n}>\delta B_{n}\right)=0$.

Theorem 16. Let $\left(\mathbb{T}_{n}\right)_{n \geqslant 1}$ be a sequence of rooted trees. Suppose that there exists a sequence $\left(a_{n}\right)_{n \geqslant 1}$ of non-negative real numbers with $\lim _{n \rightarrow \infty} a_{n}=0, \lim _{n \rightarrow \infty} n a_{n}^{1 / k}=\infty$ and such that

$$
\text { (a) } \max _{v \in \mathbb{T}_{n}} L_{n}(v)=O_{\mathrm{p}}\left(a_{n}^{-1}\right) \text {. }
$$


(b) For every $q \in \mathbb{N}, a_{n}\left(L_{n}\left(u_{1}\right), \ldots, L_{n}\left(u_{1}, \ldots, u_{q}\right)\right) \stackrel{d}{\rightarrow}\left(\zeta_{1}, \ldots, \zeta_{1}+\cdots+\zeta_{q}\right)$, as $n \rightarrow \infty$, where $\zeta_{1}, \zeta_{2} \ldots$ is a sequence of i.i.d. random variables in $\mathbb{R}_{+}$with no atom at 0 .

(c) For every $q \in \mathbb{N}, \mathbb{E}\left[\left(a_{n} L_{n}\left(u_{1}\right) \cdots a_{n} L_{n}\left(u_{q}\right)\right)^{-1 / k} \mathbb{1}_{\left\{u_{1}, \ldots, u_{q} \in \mathbb{T}_{n} \backslash\{\circ\}\right\}}\right] \rightarrow \mathbb{E}\left[\zeta_{1}^{-1 / k}\right]^{q}<$ $\infty$, as $n \rightarrow \infty$.

Then $n^{-1} a_{n}^{-1 / k} \mathcal{K}_{1}\left(\mathbb{T}_{n}\right) \stackrel{d}{\rightarrow} Z_{\zeta}$, as $n \rightarrow \infty$, where $Z_{\zeta}$ is a random variable whose law is determined entirely by its moments: $\mathbb{E}\left[Z_{\zeta}^{0}\right]=1$, and for $q \in \mathbb{N}$,

$$
\mathbb{E}\left[Z_{\zeta}^{q}\right]=q ! \int_{0}^{\infty} \int_{0}^{x_{1}} \cdots \int_{0}^{x_{q-1}} \mathbb{E}\left[\exp \left(-\frac{\zeta_{1} x_{1}^{k}+\cdots+\zeta_{q} x_{q}^{k}}{k !}\right)\right] \mathrm{d} \overleftarrow{\mathbf{x}}_{q}
$$

Proof. By the assumption (a) and Lemma 6 (in particular, the identity (17)), we see that

$$
\mathbb{E}\left[\mathcal{K}_{1}\left(\mathbb{T}_{n}\right)^{q} \mid \mathbb{T}_{n}\right]=\left(1+O\left(a_{n}^{\frac{q}{2 k}}\right)\right) q ! \sum_{v_{1}, \ldots, v_{q} \in \mathbb{T}_{n} \backslash\{0\}} \widehat{H}_{n, q}\left(\mathbf{v}_{q}\right)+Y_{q}
$$

where $\mathbf{v}_{q}=\left(v_{1}, \ldots, v_{q}\right) \in \mathbb{T}_{n}^{q}, Y_{q}:=\sum_{p=0}^{q-1} \sum_{l=0}^{p}\left(\begin{array}{l}q \\ p\end{array}\right)\left(\begin{array}{l}p \\ l\end{array}\right)(-1)^{p-l} \mathbb{E}\left[\mathcal{K}_{1}\left(\mathbb{T}_{n}\right)^{l} \mid \mathbb{T}_{n}\right]$ and

$$
\widehat{H}_{n, q}\left(\mathbf{v}_{q}\right):=\int_{0}^{\infty} \int_{0}^{x_{1}} \cdots \int_{0}^{x_{q-1}} G_{n}\left(\mathbf{v}_{q}, \mathbf{x}_{q}\right) e^{-\sum_{i=1}^{q} x_{i}} \mathrm{~d} \overleftarrow{\mathbf{x}}_{q},
$$

with $G_{n}$ defined in (14). Then we see that

$$
n^{-q} \mathbb{E}\left[\mathcal{K}_{1}\left(\mathbb{T}_{n}\right)^{q}\right]=\left(1+O\left(a_{n}^{\frac{q}{2 k}}\right)\right) q ! \mathbb{E}\left[\widehat{H}_{n, q}\left(\mathbf{u}_{q}\right) \mathbb{1}_{\left\{\mathbf{u}_{q} \in\left(\mathbb{T}_{n} \backslash\{\circ\}\right)^{q}\right\}}\right]+n^{-q} \mathbb{E}\left[Y_{q}\right]
$$

where $\mathbf{u}_{q}=\left(u_{1}, \ldots, u_{q}\right)$. Suppose that we have proven that

$$
\begin{aligned}
& a_{n}^{-q / k} \mathbb{E}\left[\widehat{H}_{n, q}\left(\mathbf{u}_{q}\right) \mathbb{1}_{\left\{\mathbf{u}_{q} \in\left(\mathbb{T}_{n} \backslash\{\circ\}\right)^{q}\right\}}\right] \\
& \rightarrow \int_{0}^{\infty} \int_{0}^{x_{1}} \cdots \int_{0}^{x_{q-1}} \mathbb{E}\left[\exp \left(-\frac{\zeta_{1} x_{1}^{k}+\cdots+\zeta_{q} x_{q}^{k}}{k !}\right)\right] \mathrm{d} \overleftarrow{\mathbf{x}}_{q} .
\end{aligned}
$$

as $n \rightarrow \infty$. Then the result follows by induction on $q \in \mathbb{N}$ together with the previous convergence.

We henceforth prove the claim in (31). From the result in (19), it is enough to check the following:

(i) The sequence $\left(a_{n}^{-q / k} \widehat{H}_{n, q}\left(\mathbf{u}_{q}\right) \mathbb{1}_{\left\{\mathbf{u}_{q} \in\left(\mathbb{T}_{n} \backslash\{\circ\}\right)^{q}\right\}}\right)_{n \geqslant 1}$ is uniformly integrable.

(ii) $a_{n}^{-q / k} \widehat{H}_{n, q}\left(\mathbf{u}_{q}\right) \mathbb{1}_{\left\{\mathbf{u}_{q} \in\left(\mathbb{T}_{n} \backslash\{\circ\}\right)^{q}\right\}} \stackrel{d}{\rightarrow} \int_{0}^{\infty} \int_{0}^{x_{1}} \cdots \int_{0}^{x_{q-1}} \exp \left(-\frac{\zeta_{1} x_{1}^{k}+\cdots+\zeta_{q} x_{q}^{k}}{k !}\right) \mathrm{d} \overleftarrow{\mathbf{x}}_{q}$, as $n \rightarrow \infty$. 
We start by showing (i). Since $\exp \left(-\left(x_{1}+\cdots+x_{q}\right)\right) \leqslant 1$ for $x_{1}, \ldots, x_{q} \in \mathbb{R}_{+}$, we have that

$$
\widehat{H}_{n, q}\left(\mathbf{u}_{q}\right) \leqslant \int_{0}^{\infty} \int_{0}^{x_{1}} \cdots \int_{0}^{x_{q-1}} G_{n}\left(\mathbf{u}_{q}, \mathbf{x}_{q}\right) \mathrm{d} \overleftarrow{\mathbf{x}}_{q}
$$

Hence after a similar computation as in the proof of the inequality (11), one obtains that there exists a constant $C_{k, q}>0$ such that

$$
a_{n}^{-q / k} \widehat{H}_{n, q}\left(\mathbf{u}_{q}\right) \mathbb{1}_{\left\{\mathbf{u}_{q} \in\left(\mathbb{T}_{n} \backslash\{\circ\}\right)^{q}\right\}} \leqslant C_{k, q} a_{n}^{-q / k}\left(L_{n}\left(u_{1}\right) \cdots L_{n}\left(u_{q}\right)\right)^{-1 / k} \mathbb{1}_{\left\{\mathbf{u}_{q} \in\left(\mathbb{T}_{n} \backslash\{\circ\}\right)^{q}\right\}} .
$$

Notice that our hypotheses (b) and (c) together with the result in (19) show that the sequence

$$
a_{n}^{-q / k}\left(\left(L_{n}\left(u_{1}\right) \cdots L_{n}\left(u_{q}\right)\right)^{-1 / k} \mathbb{1}_{\left\{\mathbf{u}_{q} \in\left(\mathbb{T}_{n} \backslash\{\circ\}\right)^{q}\right\}}\right)_{n \geqslant 1}
$$

is uniformly integrable. Hence (i) follows from [18, Theorem 5.4.5].

Finally, we verify (ii). By making the change of variables $x_{i}=a_{n}^{1 / k} w_{i}$, for $1 \leqslant i \leqslant q$, we see that

$$
\widehat{H}_{n, q}\left(\mathbf{u}_{q}\right)=a_{n}^{q / k} \int_{0}^{\infty} \int_{0}^{w_{1}} \cdots \int_{0}^{w_{q-1}} \bar{G}_{n}\left(\mathbf{u}_{q}, \mathbf{w}_{q}\right) e^{-a_{n}^{1 / k} \sum_{i=1}^{q} w_{i}} \mathrm{~d} \overleftarrow{\mathbf{w}}_{q}
$$

where $\mathbf{w}_{q}=\left(w_{1}, \ldots, w_{q}\right) \in \mathbb{R}_{+}^{q}, \overleftarrow{\mathbf{w}}_{q}=\left(w_{q}, \ldots, w_{1}\right)$, and

$$
\bar{G}_{n}\left(\mathbf{u}_{q}, \mathbf{w}_{q}\right):=\exp \left(-\frac{a_{n} D_{n}\left(u_{1}\right) w_{1}^{k}+\cdots+a_{n} D_{n}\left(u_{1}, \ldots, u_{q}\right) w_{q}^{k}}{k !}\right),
$$

with $D_{n}\left(u_{1}\right):=L_{n}\left(u_{1}\right)$ and $D_{n}\left(u_{1}, \ldots, u_{q}\right):=L_{n}\left(u_{1}, \ldots, u_{q}\right)-L_{n}\left(u_{1}, \ldots, u_{q-1}\right)$ for $q \geqslant 2$. Notice that $\mathbb{1}_{\left\{\mathbf{u}_{q} \in\left(\mathbb{T}_{n} \backslash\{\circ\}\right)^{q}\right\}} \stackrel{d}{\rightarrow} 1$, as $n \rightarrow \infty$. Thus, condition (b) implies that

$$
\bar{G}_{n}\left(\mathbf{u}_{q}, \mathbf{w}_{q}\right) \mathbb{1}_{\left\{\mathbf{u}_{q} \in\left(\mathbb{T}_{n} \backslash\{\circ\}\right)^{q}\right\}} \stackrel{d}{\rightarrow} \exp \left(-\frac{\zeta_{1} w_{1}^{k}+\cdots+\zeta_{q} w_{q}^{k}}{k !}\right), \quad \text { as } n \rightarrow \infty .
$$

By the Skorohod coupling theorem (see e.g. [27, Theorem 4.30]), we can assume that the previous convergence holds almost surely together with the convergence in condition (b). Notice that for $\varepsilon \in(0,1)$ there exists $N \in \mathbb{N}$ such that

$$
\bar{G}_{n}\left(\mathbf{v}_{q}, \mathbf{w}_{q}\right) \mathbb{1}_{\left\{\mathbf{v}_{q} \in\left(\mathbb{T}_{n} \backslash\{\circ\}\right)^{q}\right\}} \leqslant \exp \left(-(1-\varepsilon) \zeta_{1} w_{1}^{k} / k !\right), \text { for } n \geqslant N .
$$

By condition (c), notice also that the function on the right-hand side is integrable on $\left\{\mathbf{w}_{q} \in \mathbb{R}_{+}^{q}: 0 \leqslant w_{q} \leqslant \cdots \leqslant w_{1}<\infty\right\}$. Therefore, it should be clear now that (ii) follows by the dominated convergence theorem. This concludes our proof. 
The next result establishes an estimate for the mean number of $r$-records $\mathcal{K}_{r}\left(\mathbb{T}_{n}\right)$ of a general (random) rooted tree in the same spirit as in Lemma 8. Furthermore, it shows that $\mathcal{K}_{r}\left(\mathbb{T}_{n}\right)$ is of smaller order than $\mathcal{K}_{1}\left(\mathbb{T}_{n}\right)$ and hence it will not contribute (in the limit) to the distribution of the $k$-cut number $\mathcal{K}\left(\mathbb{T}_{n}\right)$. We believe as well that our methods can be used to estimate higher moments and to obtain an analogue result to Theorem 16 for $\mathcal{K}_{r}\left(\mathbb{T}_{n}\right)$. We have not attempted to do it and the estimation of the mean is enough for our purpose.

Lemma 17. Let $\left(\mathbb{T}_{n}\right)_{n \geqslant 1}$ be a sequence of rooted trees. Suppose that there exists a sequence $\left(a_{n}\right)_{n \geqslant 1}$ of non-negative real numbers with $\lim _{n \rightarrow \infty} a_{n}=0, \lim _{n \rightarrow \infty} n a_{n}=\infty$ and such that

(a) $\max _{v \in \mathbb{T}_{n}} L_{n}(v)=O_{\mathrm{p}}\left(a_{n}^{-1}\right)$.

(b) $a_{n} L_{n}\left(u_{1}\right) \stackrel{d}{\rightarrow} \zeta_{1}$, as $n \rightarrow \infty$, where $\zeta_{1}$ is a random variable in $\mathbb{R}_{+}$with no atom at 0 .

(c) For every $r \in\{1, \ldots k\}, \mathbb{E}\left[\left(a_{n} L_{n}\left(u_{1}\right)\right)^{-r / k} \mathbb{1}_{\left\{u_{1} \in \mathbb{T}_{n} \backslash\{\circ\}\right\}}\right] \rightarrow \mathbb{E}\left[\zeta_{1}^{-r / k}\right]<\infty$, as $n \rightarrow \infty$. Then, for $r \in\{1, \ldots k\}$,

$$
n^{-1} a_{n}^{-r / k} \mathbb{E}\left[\mathcal{K}_{r}\left(\mathbb{T}_{n}\right)\right] \rightarrow \frac{(k !)^{r / k} \Gamma(r / k)}{k \Gamma(r)} \mathbb{E}\left[\zeta_{1}^{-r / k}\right], \text { as } n \rightarrow \infty .
$$

Proof. By the assumption (a) and Lemma 7 (in particular, the identity (20)), we see that

$$
\mathbb{E}\left[\mathcal{K}_{r}\left(\mathbb{T}_{n}\right) \mid \mathbb{T}_{n}\right]=\left(1+O\left(a_{n}^{\frac{1}{2 k}}\right)\right) \sum_{v \in \mathbb{T}_{n} \backslash\{0\}} \int_{0}^{\infty} \frac{x^{r-1} e^{-x}}{\Gamma(r)} e^{-\frac{L_{n}(v) x^{k}}{k !}} \mathrm{d} x+o\left(n a_{n}^{r / k}\right) .
$$

Hence

$$
n^{-1} \mathbb{E}\left[\mathcal{K}_{r}\left(\mathbb{T}_{n}\right)\right]=\left(1+O\left(a_{n}^{\frac{1}{2 k}}\right)\right) \int_{0}^{\infty} \frac{x^{r-1} e^{-x}}{\Gamma(r)} \mathbb{E}\left[e^{-\frac{L_{n}\left(u_{1}\right) x^{k}}{k !}} \mathbb{1}_{\left\{u_{1} \in \mathbb{T}_{n} \backslash\{0\}\right\}}\right] \mathrm{d} x+o\left(a_{n}^{r / k}\right) .
$$

Therefore, our result follows by proving that

$$
a_{n}^{-r / k} \int_{0}^{\infty} \frac{x^{r-1} e^{-x}}{\Gamma(r)} \mathbb{E}\left[e^{-\frac{L_{n}\left(u_{1}\right) x^{k}}{k !}} \mathbb{1}_{\left\{u_{1} \in \mathbb{T}_{n} \backslash\{\circ\}\right\}}\right] \mathrm{d} x \rightarrow \int_{0}^{\infty} \frac{x^{r-1}}{\Gamma(r)} \mathbb{E}\left[e^{-\frac{\zeta_{1} x^{k}}{k !}}\right] \mathrm{d} x \text {, as } n \rightarrow \infty,
$$

where the last integral is equal to the right-hand side of (32). Note that the case $r=1$ has been proved in Theorem 16. The proof of the general case $r \in\{1, \ldots, k\}$ follows by a simple adaptation of the argument used in the proof of Theorem 16 for $q=1$ and details are left to the reader.

The next lemma provides a useful way to verify condition (c) in Theorem 16 .

Lemma 18. Let $\mathbb{T}_{n}$ be a rooted tree. Suppose that there exists a sequence $\left(a_{n}\right)_{n \geqslant 1}$ of nonnegative real numbers with $\lim _{n \rightarrow \infty} a_{n}=0, \lim _{n \rightarrow \infty} n a_{n}^{1 / k}=\infty$ and such that for every $q \in \mathbb{N}$,

$$
a_{n}\left(L_{n}\left(u_{1}\right), \cdots, L_{n}\left(u_{q}\right)\right) \stackrel{d}{\rightarrow}\left(\zeta_{1}, \ldots, \zeta_{q}\right), \quad \text { as } n \rightarrow \infty
$$


where $\zeta_{1}, \zeta_{2} \ldots$ is a sequence of $i . i . d$. random variables in $\mathbb{R}_{+}$with no atom at 0 such that $\mathbb{E}\left[\zeta_{1}^{-1 / k}\right]<\infty$. Furthermore, assume that for every $q \in \mathbb{N}$ there exists $\delta>0$ such that for all $\varepsilon \in(0, \delta)$

$$
\mathbb{E}\left[W_{i}\left(\mathbb{T}_{n}\right)\right]=o\left(n a_{n}^{q / k+1}\right), \text { uniformly on } 0 \leqslant i \leqslant \varepsilon^{1 / q} a_{n}^{-1},
$$

where $W_{i}\left(\mathbb{T}_{n}\right)$ denotes the number of vertices a depth $i \in \mathbb{Z}_{\geqslant 0}$ in $\mathbb{T}_{n}$. Then the condition (c) in Theorem 16 is satisfied

Proof. For simplicity, we introduce the notation

$$
X_{n, q}:=\left(a_{n} L_{n}\left(u_{1}\right) \cdots a_{n} L_{n}\left(u_{q}\right)\right)^{-1 / k} \mathbb{1}_{\left\{\mathbf{u}_{q} \in\left(\mathbb{T}_{n} \backslash\{\circ\}\right)^{q}\right\}}
$$

and $X_{q}:=\left(\zeta_{1} \cdots \zeta_{q}\right)^{-1 / k}$, for $n, q \in \mathbb{N}$. Consider $\delta>0$ such that for $\varepsilon \in(0, \delta)$ the property in (33) is satisfied. Define the function $\phi_{\varepsilon}: \mathbb{R}_{+} \rightarrow \mathbb{R}_{+}$given by $\phi_{\varepsilon}=0$ on $[0, \varepsilon], \phi_{\varepsilon}=1$ on $[2 \varepsilon, \infty)$, and $\phi_{\varepsilon}$ linear on $[\varepsilon, 2 \varepsilon]$. Since $\mathbb{1}_{\left\{\mathbf{u}_{q} \in\left(\mathbb{T}_{n} \backslash\{\circ\}\right)^{q}\right\}} \stackrel{d}{\rightarrow} 1$ we observe that

$$
\mathbb{E}\left[X_{n, q} \phi_{\varepsilon}\left(X_{n, q}^{-k}\right)\right] \rightarrow \mathbb{E}\left[X_{q} \phi_{\varepsilon}\left(X_{q}^{-k}\right)\right], \quad \text { as } n \rightarrow \infty \text {. }
$$

Further, we note that $\phi_{\varepsilon}\left(X_{q}^{-k}\right) \rightarrow 1$, almost surely, as $\varepsilon \rightarrow 0$. In order to show that condition (c) in Theorem 16 is fulfilled, it is enough to check that

$$
\lim _{\varepsilon \rightarrow 0} \lim _{n \rightarrow \infty} \mathbb{E}\left[\left(X_{n, q}-X_{n, q} \phi_{\varepsilon}\left(X_{n, q}^{-k}\right)\right)\right]=0 .
$$

Notice that

$$
\mathbb{E}\left[\left(X_{n, q}-X_{n, q} \phi_{\varepsilon}\left(X_{n, q}^{-k}\right)\right)\right] \leqslant \mathbb{E}\left[X_{n, q} \mathbb{1}_{\left\{X_{n, q}^{-k} \leqslant \varepsilon\right\}}\right] .
$$

Since $\left\{X_{n, q}^{-k} \leqslant \varepsilon\right\} \subseteq\left\{1 \leqslant L_{n}\left(u_{1}\right) \leqslant \varepsilon^{1 / q} a_{n}^{-1}\right\} \cap \cdots \cap\left\{1 \leqslant L_{n}\left(u_{q}\right) \leqslant \varepsilon^{1 / q} a_{n}^{-1}\right\}$, it is not difficult to see that

$$
\begin{aligned}
\mathbb{E}\left[\left(X_{n, q}-X_{n, q} \phi_{\varepsilon}\left(X_{n, q}^{-k}\right)\right)\right] & \leqslant \mathbb{E}\left[\left(\frac{1}{n} \sum_{v \in \mathbb{T}_{n} \backslash\{0\}}\left(a_{n} L_{n}(v)\right)^{-1 / k} \mathbb{1}_{\left\{L_{n}(v) \leqslant \varepsilon^{1 / q} a_{n}^{-1}\right\}}\right)^{q}\right] \\
& \leqslant \frac{1}{n} \mathbb{E}\left[\sum_{v \in \mathbb{T}_{n} \backslash\{0\}}\left(a_{n} L_{n}(v)\right)^{-q / k} \mathbb{1}_{\left\{L_{n}(v) \leqslant \varepsilon^{1 / q} a_{n}^{-1}\right\}}\right],
\end{aligned}
$$

where we have used Jensen's inequality to obtain the second inequality. Finally, by our choice of $\varepsilon$ (recall assumption (33)), we observe that

$$
\mathbb{E}\left[\left(X_{n, q}-X_{n, q} \phi_{\varepsilon}\left(X_{n, q}^{-k}\right)\right)\right] \leqslant n^{-1} a_{n}^{-q / k} \sum_{i=1}^{\left\lfloor\varepsilon^{1 / q} a_{n}^{-1}\right\rfloor} i^{-q / k} \mathbb{E}\left[W_{i}\left(\mathbb{T}_{n}\right)\right]=o(1) .
$$

This clearly implies (34) and concludes our proof. 
Similarly, we also provide a useful way to verify condition (c) in Lemma 17.

Lemma 19. Let $\mathbb{T}_{n}$ be a rooted tree. Suppose that there exists a sequence $\left(a_{n}\right)_{n \geqslant 1}$ of nonnegative real numbers with $\lim _{n \rightarrow \infty} a_{n}=0, \lim _{n \rightarrow \infty} n a_{n}=\infty$ and such that the condition (b) in Lemma 17 holds with a random variable $\zeta_{1}$ satisfying $\mathbb{E}\left[\zeta_{1}^{-r / k}\right]<\infty$ for every $r \in\{1, \ldots, k\}$. Furthermore, assume that for every $r \in\{1, \ldots, k\}$ there exists $\delta>0$ such that for all $\varepsilon \in(0, \delta)$

$$
\mathbb{E}\left[W_{i}\left(\mathbb{T}_{n}\right)\right]=o\left(n a_{n}^{r / k+1}\right), \quad \text { uniformly on } 0 \leqslant i \leqslant \varepsilon a_{n}^{-1},
$$

where $W_{i}\left(\mathbb{T}_{n}\right)$ denotes the number of vertices at depth $i \in \mathbb{Z}_{\geqslant 0}$ in $\mathbb{T}_{n}$. Then the condition (c) in Lemma 17 is fulfilled.

Proof. It should be clear that this can be shown along the lines of the proof of Lemma 18, and therefore, we omit its proof.

\subsection{Trees of logarithmic height}

Natural examples of trees that fulfil the conditions of Theorem 16 are the class of random trees with logarithmic height, i.e., trees $\mathbb{T}_{n}$ such that $\max _{v \in \mathbb{T}_{n}} d_{n}(v)=O_{\mathrm{p}}(\ln n)$. For instance, random split trees, uniform random recursive trees, scale-free random trees and mixtures of complete regular trees.

\subsubsection{Complete binary trees}

Let $\mathbb{T}_{n}^{\text {bi }}$ be a complete binary tree with $n \in \mathbb{N}$ vertices, i.e., its height is $\lfloor\ln n\rfloor$. Recall that $\mathbb{T}_{n}^{\text {bi }}$ has $2^{i}$ vertices at height $i \in\{0,1, \ldots,\lfloor\ln n\rfloor\}$, and $n-2^{\lfloor\ln n\rfloor}+1$ vertices of height $\lfloor\ln n\rfloor$, moreover, the vertices of height $\lfloor\ln n\rfloor$ have leftmost positions among the $2^{\lfloor\ln n\rfloor}$ possible ones; see, e.g., [28, Page 401]. We use the notation $\lg _{2} n=(\ln n) /(\ln 2)$ for the logarithm with base 2 of $n \in \mathbb{N}$. It should be clear that condition (a) in Theorem 16 is satisfied with $a_{n}=\left(\lg _{2} n\right)^{-1}$. Furthermore, one readily checks that $\left(\lg _{2} n\right)^{-1}\left(L_{n}\left(u_{1}\right), L_{n}\left(u_{1}, u_{2}\right)\right) \stackrel{d}{\rightarrow}(1,2)$, as $n \rightarrow \infty$. By a simple application of [5, Corollary 1], this implies that condition (b) in Theorem 16 is satisfied with $\zeta_{1} \equiv 1$. Notice that each vertex in $\mathbb{T}_{n}^{\text {bi }}$ has at most 2 children. Then it should be clear that condition (c) of Theorem 16 follows from Lemma 18 since $\mathbb{E}\left[W_{i}\left(\mathbb{T}_{n}^{\text {bi }}\right)\right] \leqslant 2^{i}$ for $i \in \mathbb{Z}_{\geqslant 0}$. Therefore, Theorem 16 implies that $n^{-1}\left(\lg _{2} n\right)^{1 / k} \mathcal{K}_{1}\left(\mathbb{T}_{n}^{\mathrm{bi}}\right) \stackrel{d}{\rightarrow} Z_{1}$, as $n \rightarrow \infty$, where $Z_{1}$ is the random variable whose law is determined entirely by its moments: $\mathbb{E}\left[Z_{1}^{0}\right]=1$, and for $q \in \mathbb{N}$,

$$
\mathbb{E}\left[Z_{1}^{q}\right]=q ! \int_{0}^{\infty} \int_{0}^{x_{1}} \cdots \int_{0}^{x_{q-1}} \exp \left(-\frac{x_{1}^{k}+\cdots+x_{q}^{k}}{k !}\right) \mathrm{d} \overleftarrow{\mathbf{x}}_{q} .
$$

It should be clear that Lemma 17 and Lemma 19 imply that

$$
\mathbb{E}\left[\mathcal{K}_{r}\left(\mathbb{T}_{n}^{\mathrm{bi}}\right)\right]=O\left(n\left(\lg _{2} n\right)^{-r / k}\right)
$$


for $r \in\{1, \ldots, k\}$. Therefore, by the identity (5) and Markov's inequality,

$$
n^{-1}\left(\lg _{2} n\right)^{1 / k} \mathcal{K}\left(\mathbb{T}_{n}^{\mathrm{bi}}\right) \stackrel{d}{\rightarrow} Z_{1}, \quad \text { as } n \rightarrow \infty .
$$

However, it follows from the next lemma that $Z_{1} \equiv(k !)^{\frac{1}{k}} \Gamma(1+1 / k)$. Therefore, we actually have

$$
n^{-1}\left(\lg _{2} n\right)^{1 / k} \mathcal{K}\left(\mathbb{T}_{n}^{\mathrm{bi}}\right) \stackrel{d}{\rightarrow} Z_{1} \equiv(k !)^{1 / k} \Gamma(1+1 / k) .
$$

Remark 20. As Theorem 1.1 of [11] shows, $\mathcal{K}\left(\mathbb{T}^{\mathrm{bi}}\right)$, after proper shifting and rescaling, also converges to a non-degenerate limit distribution with an infinite mean. Thus it is not possible to derive the result in [11] with the method of moments which we use to derive Theorem 1 for conditioned Galton-Watson trees. The same is true for split trees, random recursive trees and scale-free trees.

Lemma 21. For $q \in \mathbb{N}$, we have that

$$
q ! \int_{0}^{\infty} \int_{0}^{x_{1}} \cdots \int_{0}^{x_{q-1}} \exp \left(-\frac{x_{1}^{k}+\cdots+x_{q}^{k}}{k !}\right) \mathrm{d} \overleftarrow{\mathbf{x}}_{q}=(k !)^{q / k} \Gamma\left(1+\frac{1}{k}\right)^{q} .
$$

Proof. By making the change of variables $w_{i}=x_{i}^{k} / k$ !, for $1 \leqslant i \leqslant q$, we notice that the integral at the right-hand side of (36) is equal to

$$
q !(k !)^{q / k} \Gamma\left(1+\frac{1}{k}\right)^{q} \int_{0}^{\infty} \int_{0}^{w_{1}} \cdots \int_{0}^{w_{q-1}} \prod_{i=1}^{q} \frac{e^{-w_{i}} w_{i}^{\frac{1}{k}-1}}{\Gamma(1 / k)} \mathrm{d} \overleftarrow{\mathbf{w}}_{q}=(k !)^{q / k} \Gamma\left(1+\frac{1}{k}\right)^{q} .
$$

To see the last identity, we notice that the integral at the left-hand side is simply the probability that $G_{1} \geqslant G_{2} \geqslant \ldots \geqslant G_{q}$, where $G_{1}, \ldots, G_{q}$ are independent $\operatorname{Gamma}(1 / k, 1)$ random variables, which is equal to $1 / q$ ! since each order of $G_{1}, \ldots, G_{q}$ is equally likely.

\subsubsection{Split trees}

The class of random split trees was first introduced by Devroye [13] to encompass many families of trees that are frequently used in algorithm analysis, e.g., binary search trees and tries. Its exact construction is somewhat lengthy and we refer readers to either the original algorithmic definition in $[13,21]$ or the more probabilistic version in $[10$, Section 2]. Informally speaking, a split tree $\mathbb{T}_{n}^{\mathrm{sp}}$ is constructed by first distributing $n \in \mathbb{N}$ balls among the vertices of an infinite $b$-ary tree $(b \in \mathbb{N} \backslash\{1\})$ and then removing all subtrees without balls. Each vertex in the infinite $b$-ary tree is given a random non-negative split vector $\mathcal{V}=\left(V_{1}, \ldots, V_{b}\right)$ such that $\sum_{i=1}^{b} V_{i}=1$ and $V_{i} \geqslant 0$, drawn independently from the same distribution. These vectors affect how balls are distributed. In the study of split-trees, the following condition of $\mathcal{V}$ is often assumed (see, e.g., Holmgren [21]):

Condition A. The split vector $\mathcal{V}$ is permutation invariant. Moreover, $\mathbb{P}\left(V_{1}=1\right)=$ $\mathbb{P}\left(V_{1}=0\right)=0$, and that $-\log \left(V_{1}\right)$ is non-lattice. 
Set $\mu:=b \mathbb{E}\left[-V_{1} \ln V_{1}\right] \in(0, \ln b)$. Devroye [13] showed that $\max _{v \in \mathbb{T}_{n}^{\mathrm{sp}}} d_{n}(v)=O_{\mathrm{p}}(\ln n)$, that is, condition (a) in Theorem 16 with $a_{n}=\mu(\ln n)^{-1}$. Berzunza et al. [7, Lemma 5 and Corollary 1] have shown that $\mu(\ln n)^{-1}\left(L_{n}\left(u_{1}\right), L_{n}\left(u_{1}, u_{2}\right)\right) \stackrel{d}{\rightarrow}(1,2)$, as $n \rightarrow \infty$. By a simple application of [5, Corollary 1], this implies that condition (b) in Theorem 16 is satisfied with $\zeta_{1} \equiv 1$. Notice that each vertex in $\mathbb{T}_{n}^{\text {sp }}$ has at most $b$ children. Then it should be clear that condition (c) of Theorem 16 follows from Lemma 18 since $\mathbb{E}\left[W_{i}\left(\mathbb{T}_{n}^{\mathrm{sp}}\right)\right] \leqslant b^{i}$ for $i \in \mathbb{Z}_{\geqslant 0}$. Therefore, Theorem 16 implies that $\mu^{-1 / k} n^{-1}(\ln n)^{1 / k} \mathcal{K}_{1}\left(\mathbb{T}_{n}^{\mathrm{sp}}\right) \stackrel{d}{\rightarrow} Z_{1}$, as $n \rightarrow \infty$, where $Z_{1}$ is the random variable whose law is determined entirely by its moments given in (36). Furthermore, Lemma 17 and Lemma 19 imply that $\mathbb{E}\left[\mathcal{K}_{r}\left(\mathbb{T}_{n}^{\mathrm{sp}}\right)\right]=O\left(n(\ln n)^{-r / k}\right)$ for $r \in\{1, \ldots, k\}$. Therefore, by the identity (5) and the Markov's inequality,

$$
\mu^{-1 / k} n^{-1}(\ln n)^{1 / k} \mathcal{K}_{1}\left(\mathbb{T}_{n}^{\mathrm{sp}}\right) \stackrel{d}{\rightarrow} Z_{1} \equiv(k !)^{1 / k} \Gamma(1+1 / k) .
$$

\subsubsection{Uniform random recursive trees}

A uniform random recursive tree $\mathbb{T}_{n}^{\mathrm{rr}}$ is a random tree of $n \in \mathbb{N}$ vertices constructed recursively as follows: let $\mathbb{T}_{1}^{\text {rr }}$ be the tree of a single vertex labelled 1 , given $\mathbb{T}_{n-1}^{\text {rr }}$, choose a vertex in $\mathbb{T}_{n-1}^{\text {rr }}$ uniformly at random and attach a vertex labelled $n$ to the selected vertex as its child, which give $\mathbb{T}_{n}^{\mathrm{rr}}$. The uniform random recursive tree is one of the most studied random tree models. They appear for instance as simple epidemic models, or in computer science as data structures. We refer to [15, Chapter 6] for background. Theorem 6.32 in [15] shows that $\max _{v \in \mathbb{T}_{n}^{\mathrm{rr}}} d_{n}(v)=O_{\mathrm{p}}(\ln n)$, that is, condition (a) in Theorem 16 is satisfied with $a_{n}=(\ln n)^{-1}$. From the results of Dobrow [14] (see also [15, Section 2.5.5]), it is not difficult to see that $(\ln n)^{-1}\left(L_{n}\left(u_{1}\right), L_{n}\left(u_{1}, u_{2}\right)\right) \stackrel{d}{\rightarrow}(1,2)$, as $n \rightarrow \infty$. By a simple application of [5, Corollary 1], this implies that condition (b) in Theorem 16 is satisfied with $\zeta_{1} \equiv 1$. By [17, Equation (11)],

$$
\mathbb{E}\left[W_{i}\left(\mathbb{T}_{n}^{\mathrm{rr}}\right)\right]=\frac{(\ln n)^{i}}{\Gamma(1+1 /(\ln n)) i !}(1+O(1 /(\ln n)))
$$

uniformly for $n \geqslant 3$ and $1 \leqslant i \leqslant K \ln n$, for all $K \geqslant 1$. Then it should be clear that condition (c) of Theorem 16 follows from Lemma 18. Therefore, Theorem 16 implies that $n^{-1}(\ln n)^{1 / k} \mathcal{K}_{1}\left(\mathbb{T}_{n}^{\mathrm{rr}}\right) \stackrel{d}{\rightarrow} Z_{1}$, as $n \rightarrow \infty$, where $Z_{1}$ is the random variable whose law is entirely determined by its moments given in (36). Furthermore, Lemma 17 and Lemma 19 imply that $\mathbb{E}\left[\mathcal{K}_{r}\left(\mathbb{T}_{n}^{\mathrm{rr}}\right)\right]=O\left(n(\ln n)^{-r / k}\right)$ for $r \in\{1, \ldots, k\}$. Therefore, by the identity (5) and the Markov's inequality,

$$
n^{-1}(\ln n)^{1 / k} \mathcal{K}_{1}\left(\mathbb{T}_{n}^{\mathrm{rr}}\right) \stackrel{d}{\rightarrow} Z_{1} \equiv(k !)^{1 / k} \Gamma(1+1 / k) .
$$

\subsubsection{Scale-free random trees}

Scale-free random trees form a family of random trees that grow following a preferential attachment algorithm, and are commonly used to model complex real-world networks; see Móri [32]. A scale-free random tree $\mathbb{T}_{n}^{\text {sf }}$ is a random tree of $n \in \mathbb{N}$ vertices constructed 
recursively as follows: Fix a parameter $\alpha \in(-1, \infty)$, and start from the tree $\mathbb{T}_{1}^{\text {sf }}$ that consists in a single edge connecting the vertices labelled 1 and 2 . Suppose that $T_{n}^{\text {sf }}$ has

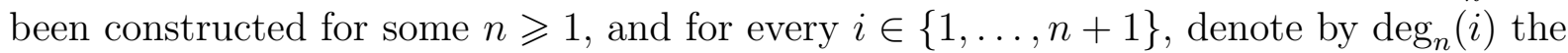
degree of the vertex $i$ in $T_{n}^{\mathrm{sf}}$. Then conditionally given $T_{n}^{\mathrm{sf}}, T_{n+1}^{\mathrm{sf}}$ is built by adding an edge between the new vertex $n+2$ and a vertex $v_{n}$ in $T_{n}^{\mathrm{sf}}$ chosen at random according to the law

$$
\mathbb{P}\left(v_{n}=i \mid T_{n}^{\mathrm{sf}}\right)=\frac{\operatorname{deg}_{n}(i)+\alpha}{2 n+\alpha(n+1)}, \quad i \in\{1, \ldots, n+1\} .
$$

The standard preferential attachment tree (also known as plane-oriented recursive tree) was made popular by Barabási and Albert [4] and it corresponds to the choice of $\alpha=0$. On the other hand, if one lets $\alpha \rightarrow \infty$, then the algorithm yields a uniform random recursive tree. Janson [26] showed that scale-free random trees can also be viewed as split trees with the branching factor $b=\infty$.

Pittel [35] showed that $\max _{v \in \mathbb{T}_{n}^{\text {sf }}} d_{n}(v)=O_{\mathrm{p}}(\ln n)$, that is, condition (a) in Theorem 16 is satisfied with $a_{n}=(\beta \ln n)^{-1}$, where $\beta:=(1+\alpha) /(2+\alpha)$. From the results of Borovkov and Vatutin [9] (see the bibliography therein for further references), it is not difficult to see that $(\beta \ln n)^{-1}\left(L_{n}\left(u_{1}\right), L_{n}\left(u_{1}, u_{2}\right)\right) \stackrel{d}{\rightarrow}(1,2)$, as $n \rightarrow \infty$. By a simple application of [5, Corollary 1], this implies that condition (b) in Theorem 16 is satisfied with $\zeta_{1} \equiv 1$. Hwang $[22$, Equation 8] showed that, for $\alpha=0$, i.e., for the standard preferential attachment tree,

$$
\mathbb{E}\left[W_{i}\left(\mathbb{T}_{n}^{\mathrm{sf}}\right)\right]=\frac{\sqrt{\pi n} 2^{1-i}(\ln n)^{i-1}}{\Gamma(i)(2 i /(\ln n)+1) \Gamma(i /(\ln n)+1)}(1+O(1 /(\ln n))),
$$

uniformly for $1 \leqslant i \leqslant K \ln n$ for all $K \geqslant 1$. Thus by an argument similar to that for uniform random recursive trees, we have for $\alpha=0$,

$$
2^{-1 / k} n^{-1}(\ln n)^{1 / k} \mathcal{K}\left(\mathbb{T}_{n}^{\mathrm{st}}\right) \stackrel{d}{\rightarrow} Z_{1} \equiv(k !)^{1 / k} \Gamma(1+1 / k) .
$$

Open problem. To apply Theorem 16 to general scale-free trees, we need an estimate of $\mathbb{E}\left[W_{i}\left(\mathbb{T}_{n}^{\mathrm{sf}}\right)\right]$ for all $\alpha>-1$, which is currently missing in the literature. Thus we leave it as an open problem that an estimation similar to (38) holds for all $\alpha>-1$. This would imply that the convergence in (39) holds for all scale-free trees.

Remark 22. In all previous examples of Section 4.3, the limit distributions found here are all degenerate. However, we conjecture that another normalization should yield to non-degenerate limits. This is known to be the case, when $k=1$, for complete binary trees (Janson [24]), recursive trees (Drmota et al. [16], Iksanov and Möhle [23]), binary search trees (Holmgren [19]) and split trees (Holmgren [20]). In the general case $k \geqslant 1$, Cai and Holmgren [11] obtained also a weak limit theorem in the case of complete binary trees suggesting that our conjecture must be true.

\subsubsection{Mixture of regular trees}

Our next example provides a method to build trees that fulfill the conditions of Theorem 16 where the random variables $\zeta_{1}, \zeta_{2}, \ldots$ in the hypotheses are not constants. Basically, 
the procedure consists of gluing trees which satisfy the assumptions of Theorem 16 . In this example, we consider a mixture of complete regular trees but one may consider other families of trees as well. For a fixed integer $m \geqslant 1$, let $\left(d_{i}\right)_{i=1}^{m}$ denote a positive sequence of integers. Next, for $i=1, \ldots, m$, let $h_{i}(n): \mathbb{R}_{+} \rightarrow \mathbb{R}_{+}$be a function with $\lim _{n \rightarrow \infty} h_{i}(n)=\infty$. Let $T_{n_{i}}^{\left(d_{i}\right)}$ be a complete $d_{i}$-regular tree with height $\left\lfloor h_{i}(n)\right\rfloor$. Since there are $d_{i}^{j}$ vertices at distance $j=0,1, \ldots,\left\lfloor h_{i}(n)\right\rfloor$ from the root, its size is given by

$$
n_{i}=n_{i}(n)=d_{i}\left(d_{i}^{\left\lfloor h_{i}(n)\right\rfloor}-1\right) /\left(d_{i}-1\right) .
$$

In particular, one can check that each tree $T_{n_{i}}^{\left(d_{i}\right)}$ fulfills the assumptions in Theorem 16 with $a_{n}=\ln n_{i}$ and $\zeta_{1}=\left(\ln d_{i}\right)^{-1}$; note that condition (c) in Theorem 16 follows from Lemma 18 and the fact that the number of descendants of each vertex is bounded. Now imagine that we merge all the $m$ regular trees into one common root. This leads us to a new tree $T_{n}^{(d)}$ of size $n=\sum_{i=1}^{m} n_{i}+1-m$. Assume further that $n_{1} \sim n_{2} \sim \cdots \sim n_{m}$, as $n \rightarrow \infty$. Then, we observe that the probability that a vertex of $T_{n}^{(d)}$ chosen uniformly at random belongs to the tree $T_{n_{i}}^{\left(d_{i}\right)}$ converges when $n \rightarrow \infty$ to $1 / m$. Then, one readily checks that this new tree satisfies the hypotheses in Theorem 16 with $a_{n}=\ln n$ and $\zeta_{1}, \zeta_{2}, \ldots$ are i.i.d. random variables uniformly distributed in the set $\left\{1 / \ln d_{1}, \ldots, 1 / \ln d_{m}\right\}$. To see this, note that the probability that a uniform chosen vertex of $T_{n}^{(d)}$ belongs to $T_{n_{i}}^{\left(d_{i}\right)}$ converges to $1 / m$.

Acknowledgements. The authors thank the referee for suggestions which improved the exposition of the paper.

\section{References}

[1] L. Addario-Berry, N. Broutin, and C. Holmgren, Cutting down trees with a Markov chainsaw, Ann. Appl. Probab. 24 (2014), no. 6, 2297-2339. MR 3262504

[2] D. Aldous, The continuum random tree. II. An overview, Stochastic Analysis (Durham, 1990), London Math. Soc. Lecture Note Ser., vol. 167, Cambridge Univ. Press, Cambridge, 1991, pp. 23-70. MR 1166406

[3] D. Aldous, The continuum random tree. III, Ann. Probab. 21 (1993), no. 1, 248-289. MR 1207226

[4] A.-L. Barabási and R. Albert, Emergence of Scaling in Random Networks, Science 286 (1999), no. 5439, 509-512 (English).

[5] J. Bertoin, Almost giant clusters for percolation on large trees with logarithmic heights, J. Appl. Probab. 50 (2013), no. 3, 603-611 (English).

[6] J. Bertoin and G. Miermont, The cut-tree of large Galton-Watson trees and the Brownian CRT, Ann. Appl. Probab. 23 (2013), no. 4, 1469-1493. MR 3098439

[7] G. Berzunza, X. Shi Cai, and C. Holmgren, The asymptotic non-normality of the giant cluster for percolation on random split trees, arXiv e-prints (2019), arXiv: 1902.08109. 
[8] R. M. Blumenthal, Excursions of Markov processes, Probability and Its Applications, Birkhäuser Boston, Inc., Boston, MA, 1992. MR 1138461

[9] K. A. Borovkov and V. A. Vatutin, On the asymptotic behaviour of random recursive trees in random environments, Adv. in Appl. Probab. 38 (2006), no. 4, 1047-1070. MR 2285693

[10] N. Broutin and C. Holmgren, The total path length of split trees, Ann. Appl. Probab. 22 (2012), no. 5, 1745-1777. MR 3025680

[11] X. S. Cai and C. Holmgren, Cutting resilient networks - complete binary trees, The Electronic Journal of Combinatorics 26 (2019), no. 4, \#P4.43 (English).

[12] X. S. Cai, C. Holmgren, L. Devroye, and F. Skerman, $k$-cut on paths and some trees, Electron. J. Probab. 24 (2019), 22 pp.

[13] L. Devroye, Universal limit laws for depths in random trees, SIAM J. Comput. 28 (1999), no. 2, 409-432. MR 1634354

[14] R. P. Dobrow, On the distribution of distances in recursive trees, J. Appl. Probab. 33 (1996), no. 3, 749-757. MR 1401472

[15] M. Drmota, Random trees, SpringerWienNewYork, Vienna, 2009. MR 2484382

[16] M. Drmota, A. Iksanov, M. Moehle, and U. Roesler, A limiting distribution for the number of cuts needed to isolate the root of a random recursive tree, Random Structures Algorithms 34 (2009), no. 3, 319-336. MR 2504401

[17] M. Fuchs, H.-K. Hwang, and R. Neininger, Profiles of random trees: Limit theorems for random recursive trees and binary search trees, Algorithmica 46 (2006), no. 3-4, 367-407. MR 2291961

[18] A. Gut, Probability: A graduate course, second ed., Springer Texts in Statistics, Springer, New York, 2013. MR 2977961

[19] C. Holmgren, Random records and cuttings in binary search trees, Combin. Probab. Comput. 19 (2010), no. 3, 391-424. MR 2607374

[20] C. Holmgren, A weakly 1-stable distribution for the number of random records and cuttings in split trees, Adv. in Appl. Probab. 43 (2011), no. 1, 151-177. MR 2761152

[21] C. Holmgren, Novel characteristics of split trees by use of renewal theory, Electron. J. Probab. 17 (2012), 27 pp.

[22] H.-K. Hwang, Profiles of random trees: Plane-Oriented recursive trees, Random Structures \& Algorithms 30 (2007), no. 3, 380-413 (English).

[23] A. Iksanov and M. Möhle, A probabilistic proof of a weak limit law for the number of cuts needed to isolate the root of a random recursive tree, Electron. Comm. Probab. 12 (2007), 28-35. MR 2407414

[24] S. Janson, Random records and cuttings in complete binary trees, Mathematics and Computer Science. III, Trends Math., Birkhäuser, Basel, 2004, pp. 241-253. MR 2090513 
[25] S. Janson, Random cutting and records in deterministic and random trees, Random Structures Algorithms 29 (2006), no. 2, 139-179. MR 2245498

[26] S. Janson, Random Recursive Trees and Preferential Attachment Trees are Random Split Trees, Combinator. Probab. Comp. 28 (2019), no. 1, 81-99 (English).

[27] O. Kallenberg, Foundations of modern probability, second ed., Probability and Its Applications (New York), Springer-Verlag, New York, 2002. MR 1876169

[28] D. E. Knuth, The art of computer programming, volume 1 (3rd ed.): Fundamental algorithms, Addison Wesley Longman Publishing Co., Inc., Redwood City, CA, USA, 1997.

[29] J.-F. Marckert and A. Mokkadem, The depth first processes of Galton-Watson trees converge to the same Brownian excursion, Ann. Probab. 31 (2003), no. 3, 1655-1678. MR 1989446

[30] A. Meir and J. W. Moon, Cutting down random trees, J. Austral. Math. Soc. 11 (1970), 313-324. MR 0284370

[31] A. Meir and J. Moon, Cutting down recursive trees, Mathematical Biosciences 21 (1974), no. 3, 173-181.

[32] T. F. Móri, On random trees, Studia Sci. Math. Hungar. 39 (2002), no. 1-2, 143-155. MR 1909153

[33] A. Panholzer, Destruction of recursive trees, Mathematics and Computer Science. III, Trends Math., Birkhäuser, Basel, 2004, pp. 267-280. MR 2090518

[34] A. Panholzer, Cutting down very simple trees, Quaest. Math. 29 (2006), no. 2, 211227. MR 2233368

[35] B. Pittel, Note on the heights of random recursive trees and random m-ary search trees, Random Structures Algorithms 5 (1994), no. 2, 337-347. MR 1262983

[36] D. Revuz and M. Yor, Continuous martingales and Brownian motion, third ed., Grundlehren Der Mathematischen Wissenschaften [Fundamental Principles of Mathematical Sciences], vol. 293, Springer-Verlag, Berlin, 1999. MR 1725357 